\title{
Performance analysis of a GI-Geo-1 buffer with a preemptive resume priority scheduling discipline
}

\author{
Joris Walraevens*, Bart Steyaert and Herwig Bruneel \\ SMACS Research Group \\ Ghent University, Vakgroep TELIN (TW07) \\ Sint-Pietersnieuwstraat 41, B-9000 Gent, Belgium.
}

Phone: 0032-9-2648902

Fax: 0032-9-2644295

E-mail: jw@telin.rug.ac.be

\begin{abstract}
In this paper, we analyze a discrete-time $G I-G e o-1$ preemptive resume priority queue. We consider two classes of packets which have to be served, where one class has preemptive resume priority over the other. We show that the use of generating functions is beneficial for analyzing the system contents and packet delay of both classes. Moments and (approximate) tail probabilities of system contents and packet delay are calculated. The influence of the priority scheduling is shown by some numerical examples.
\end{abstract}

Keywords - queueing, discrete-time, preemptive priority scheduling

\section{Introduction}

In recent years, there has been much interest devoted to incorporating multimedia applications in IP networks. Different types of traffic need different QoS standards, but share the same network resources, such as buffers and bandwidth. For real-time applications, it is important that mean delay and delay-jitter are bounded, while for non real-time applications, the Loss Ratio (LR) is the restrictive quantity.

${ }^{*}$ corresponding author 
In general, one can distinguish two priority strategies, which will be referred to as Delay priority and Space priority. Delay priority schemes attempt to guarantee acceptable delay boundaries to delay-sensitive traffic (such as voice/video). This can be achieved by giving it Head-Of-Line (HOL) priority over non-delay-sensitive traffic, and/or by sharing access to the server among the various traffic classes in such a way so that each can meet its own specific delay requirements. Several types of Delay priority (or scheduling) schemes (such as Weighted-Round-Robin (WRR), Weighted-Fair-Queueing (WFQ)) have been proposed and analyzed, each with their own specific algorithmic and computational complexity (see e.g. [11,14] and the references therein). On the other hand, Loss priority schemes attempt to minimize the packet loss of loss-sensitive traffic (such as data). An overview and classification of some Loss priority (or discarding) strategies can be found in $[3,11]$.

In this paper, we will focus on the effect of a specific type of Delay priority schemes, i.e., we will analyze a queueing system with a preemptive resume priority scheduling discipline. We assume that delay-sensitive traffic has preemptive priority over delay-insensitive traffic, i.e., when the server of the queueing system becomes empty, a packet of delay-sensitive traffic, when available, will always be scheduled next. In the remaining, we will refer to the delay-sensitive and delay-insensitive traffic as high and low priority traffic respectively. Newly arriving high priority traffic interrupts the transmission of a low priority packet that has already commenced, and the interrupted low priority packet can resume its transmission when all the high priority traffic has left the system, i.e., the part of the packet that was already transmitted before the interruption by high priority packets, does not have to be retransmitted.

In the literature, there have been a number of contributions with respect to queues with a priority scheduling discipline. An overview of some basic priority queueing models can be found in Jaiswal [7], Miller [13], Takacs [16] and Takagi [17] and the references therein. Khamisy et al. [8], Laevens et al. [10], Takine et al. [19] and Walraevens et al. [20] have studied discrete-time priority queues with deterministic service times equal to one slot. Khamisy [8] analyses the system contents of the different classes, for a queue fed by a two-state Markov modulated arrival process. Laevens [10] analyses the system contents and cell delay in the case of a multiserver queue. In Takine [19], the system contents and the delay for Markov modulated high priority arrivals and geometrically distributed low priority arrivals are presented. Walraevens [20] studies the system contents and cell delay, in the special case of an output queueing switch with Bernoulli arrivals. All these models 
have a packet transmission time of a single slot in common. Furthermore, preemptive resume priority queues have been analysed in Machihara [12], Sandhu [15] and Takine et al. [18]. Machihara [12] analyzes waiting times when high priority arrivals are distributed according to a MAP process. Sandhu [15] analyses a preemptive resume priority system where the high priority packets cannot be stored in the queue. Takine [18] studies the waiting times of customers arriving to a queue according to independent MAP processes.

In this paper, we analyze the system contents and packet delay of high and low priority traffic in a discrete-time single-server buffer with a preemptive resume priority scheduling discipline and per-slot i.i.d. arrivals. The transmission times of the packets generated by both types are assumed to be geometrically distributed (with class-dependent parameters). This is mainly done to make the analysis tractable, since the geometrical distribution has the well-known memoryless property. A negative side-effect is of course that, if transmission times of some types of customers are not geometric or arrivals are not i.i.d., the analysis presented in this paper can merely be used as an approximation. However, even then some important approximate performance measures that are practically useful can be calculated, and some important qualitative conclusions can be drawn from the analysis of this model.

As far as the model is concerned, the main difference with the articles involved with HOL priority queues listed above is that the arrival processes of the different types of cells are not mutually independent. This type of arrival process occurs for instance in a multiclass output-queueing router/switch. Therefore the different classes can not be analyzed separately (i.e., as a model with server interruptions for low priority cells, see e.g. [5]), which complicates the analysis.

We will furthermore demonstrate that an analysis based on generating functions is extremely suitable for modelling this type of buffers with a priority scheduling discipline. From these generating functions, expressions for some interesting performance measures - such as means, variances and approximate tail probabilities of system contents and packet delay - can be calculated. Determining the tail behavior of the system contents and packet delay is one of the main contributions of the paper. Although these are important quantities in the evaluation of the Quality of Service (QoS) of high and low priority packet streams, this has received only little attention up till now. We will also show that the distribution of the system contents and packet delay of low priority packets not necessarily has a geometric asymptotic behavior.

The remainder of this paper is structured as follows. In the following section, 
we present the mathematical model. In sections 3 and 4 , we will then analyze the steady-state system contents and packet delay of both classes. In sections 5 and 6, we calculate expressions for some moments and approximate tail probabilities respectively of the system contents and packet delay of both classes. Some numerical examples are treated in section 7. Finally, some conclusions are formulated in section 8.

\section{Mathematical model}

We consider a discrete-time single-server system with infinite bufferspace. Time is assumed to be slotted. There are two types of packets arriving to the system, namely packets of class 1 and packets of class 2. The number of arrivals of class $j$ during slot $k$ are independent and identically distributed (i.i.d.) and are denoted by $a_{j, k}$ $(j=1,2)$. Their joint probability mass distribution is defined as

$$
a(m, n) \triangleq \operatorname{Prob}\left[a_{1, k}=m, a_{2, k}=n\right] .
$$

Note that the number of arrivals of both classes during one slot can be correlated. The joint probability generating function (pgf) of $a_{1, k}$ and $a_{2, k}$ is defined as

$$
A\left(z_{1}, z_{2}\right) \triangleq E\left[z_{1}^{a_{1, k}} z_{2}^{a_{2, k}}\right]=\sum_{m=0}^{\infty} \sum_{n=0}^{\infty} a(m, n) z_{1}^{m} z_{2}^{n}
$$

The marginal pgf's of the number of per-slot arrivals of class $j$ are denoted by $A_{j}(z)$ $(j=1,2)$ and are given by $A(z, 1)$ and $A(1, z)$ respectively. The total number of arrivals during slot $k$ is denoted by $a_{T, k} \triangleq a_{1, k}+a_{2, k}$ and its pgf is given by $A_{T}(z)=A(z, z)$. We will furthermore denote the mean arrival rate of class $j$ packets during a slot by $\lambda_{j} \triangleq E\left[a_{j, k}\right]=A_{j}^{\prime}(1)(j=1,2)$ and the mean total arrival rate by $\lambda_{T} \triangleq \lambda_{1}+\lambda_{2}$.

The service times of class $j$ packets are assumed to be i.i.d. and geometrically distributed with parameter $\beta_{j}(j=1,2)$. Their pgf's are thus given by

$$
S_{j}(z) \triangleq \frac{\left(1-\beta_{j}\right) z}{1-\beta_{j} z}
$$

with $j=1,2$.

The class 1 packets are assumed to have preemptive resume priority over the class 2 packets and within one class the scheduling is FCFS. Due to the priority scheduling mechanism, it is as if class 1 packets (the high priority packets) are stored in front 
of class 2 packets (the low priority packets) in the queue. So, if there are any class 1 packets in the queue when the server becomes idle, the one with the longest elapsed waiting time will be served next. If, on the other hand, no class 1 packets are present in the queue at that moment, the class 2 packet with the longest elapsed waiting time, if any, will be served next. Since the priority scheduling is preemptive resume, service of a low priority packet will be interrupted by newly arriving high priority packets, and an interrupted low priority packet will transmit its not-yet-transmitted part after all high priority packets have left the system. The mean service time of a class $j$ packet, i.e., the mean time a class $j$ packet stays in the server is given by $\mu_{j}=\frac{1}{1-\beta_{j}}$. Finally, the load offered by class $j$ packets is given by $\rho_{j} \triangleq \lambda_{j} \mu_{j}$. The total load offered to the queueing system is then given by $\rho_{T} \triangleq \rho_{1}+\rho_{2}$. We assume a stable system, i.e., $\rho_{T}<1$.

\section{System contents}

We denote the system contents of class $j$ packets at the beginning of slot $k$ by $u_{j, k}$ $(j=1,2)$, i.e., at the beginning of slot $k$ there are $u_{j, k}$ class $j$ packets in the system. Their joint pgf is defined as

$$
U_{k}\left(z_{1}, z_{2}\right) \triangleq E\left[z_{1}^{u_{1, k}} z_{2}^{u_{2, k}}\right]
$$

Clearly the set $\left\{u_{1, k}, u_{2, k}\right\}$ forms a Markov chain, since the arrival process is i.i.d. and the service times are geometrically distributed. The following system equations can be established:

1. If $u_{1, k}=u_{2, k}=0$ :

$$
\begin{aligned}
& u_{1, k+1}=a_{1, k} \\
& u_{2, k+1}=a_{2, k},
\end{aligned}
$$

i.e., the only packets present in the system at the beginning of slot $k+1$ are the packets that arrived during the previous slot.

2. If $u_{1, k}=0, u_{2, k}>0$ :

$$
\begin{aligned}
& u_{1, k+1}=a_{1, k} \\
& u_{2, k+1}= \begin{cases}u_{2, k}+a_{2, k} & \text { with probability } \beta_{2} \\
u_{2, k}+a_{2, k}-1 & \text { with probability }\left(1-\beta_{2}\right),\end{cases}
\end{aligned}
$$


i.e., the class 2 packet in service stays in the system (not necessarily in the server) with probability $\beta_{2}$ and leaves the system at the end of slot $k$ with probability $1-\beta_{2}$.

3. If $u_{1, k}>0$ :

$$
\begin{aligned}
& u_{1, k+1}= \begin{cases}u_{1, k}+a_{1, k} & \text { with probability } \beta_{1} \\
u_{1, k}+a_{1, k}-1 & \text { with probability }\left(1-\beta_{1}\right)\end{cases} \\
& u_{2, k+1}=u_{2, k}+a_{2, k},
\end{aligned}
$$

i.e., the class 1 packet in service stays in the system with probability $\beta_{1}$ and leaves the system at the end of slot $k$ with probability $1-\beta_{1}$.

Using these system equations, we can derive a relation between $U_{k}\left(z_{1}, z_{2}\right)$ and $U_{k+1}\left(z_{1}, z_{2}\right)$. In the remainder, we define $\mathrm{E}[X\{Y\}]$ as $\mathrm{E}[X \mid Y] \operatorname{Prob}[Y]$. We proceed as follows, taking into account the statistical independence of the random variables $\left(u_{1, k}, u_{2, k}\right)$ and $\left(a_{1, k}, a_{2, k}\right)$ :

$$
\begin{aligned}
U_{k+1}\left(z_{1}, z_{2}\right) \triangleq & \mathrm{E}\left[z_{1}^{u_{1, k+1}} z_{2}^{u_{2, k+1}}\right] \\
= & \mathrm{E}\left[z_{1}^{a_{1, k}} z_{2}^{a_{2, k}}\left\{u_{1, k}=u_{2, k}=0\right\}\right] \\
& +\beta_{2} \mathrm{E}\left[z_{1}^{a_{1, k}} z_{2}^{u_{2, k}+a_{2, k}}\left\{u_{1, k}=0, u_{2, k}>0\right\}\right] \\
& +\left(1-\beta_{2}\right) \mathrm{E}\left[z_{1}^{a_{1, k}} z_{2}^{u_{2, k}+a_{2, k}-1}\left\{u_{1, k}=0, u_{2, k}>0\right\}\right] \\
& +\beta_{1} \mathrm{E}\left[z_{1}^{u_{1, k}+a_{1, k}} z_{2}^{u_{2, k}+a_{2, k}}\left\{u_{1, k}>0\right\}\right] \\
& +\left(1-\beta_{1}\right) \mathrm{E}\left[z_{1}^{u_{1, k}+a_{1, k}-1} z_{2}^{u_{2, k}+a_{2, k}}\left\{u_{1, k}>0\right\}\right] \\
= & A\left(z_{1}, z_{2}\right) \operatorname{Prob}\left[u_{1, k}=u_{2, k}=0\right] \\
& +A\left(z_{1}, z_{2}\right)\left(\beta_{2}+\frac{1-\beta_{2}}{z_{2}}\right) \mathrm{E}\left[z_{2}^{u_{2, k}}\left\{u_{1, k}=0, u_{2, k}>0\right\}\right] \\
& +A\left(z_{1}, z_{2}\right)\left(\beta_{1}+\frac{1-\beta_{1}}{z_{1}}\right) \mathrm{E}\left[z_{1}^{u_{1, k}} z_{2}^{u_{2, k}}\left\{u_{1, k}>0\right\}\right] \\
= & A\left(z_{1}, z_{2}\right) U_{k}(0,0)+A\left(z_{1}, z_{2}\right)\left(\beta_{2}+\frac{1-\beta_{2}}{z_{2}}\right)\left[U_{k}\left(0, z_{2}\right)-U_{k}(0,0)\right] \\
& +A\left(z_{1}, z_{2}\right)\left(\beta_{1}+\frac{1-\beta_{1}}{z_{1}}\right)\left[U_{k}\left(z_{1}, z_{2}\right)-U_{k}\left(0, z_{2}\right)\right]
\end{aligned}
$$

We assume the system is stable and as a result $U_{k}\left(z_{1}, z_{2}\right)$ and $U_{k+1}\left(z_{1}, z_{2}\right)$ converge both to a common steady state value $U\left(z_{1}, z_{2}\right)$. By taking the $k \rightarrow \infty$ limit of equation (1), we obtain:

$$
\begin{aligned}
U\left(z_{1}, z_{2}\right)= & \frac{A\left(z_{1}, z_{2}\right)}{z_{2}\left[\left(1-\beta_{1} A\left(z_{1}, z_{2}\right)\right) z_{1}-\left(1-\beta_{1}\right) A\left(z_{1}, z_{2}\right)\right]}\left[z_{1}\left(1-\beta_{2}\right)\left(z_{2}-1\right) U(0,0)\right. \\
& \left.+\left(\left(1-\beta_{2}\right) z_{1}-\left(1-\beta_{1}\right) z_{2}+\left(\beta_{2}-\beta_{1}\right) z_{1} z_{2}\right) U\left(0, z_{2}\right)\right]
\end{aligned}
$$


It now remains for us to determine the unknown function $U\left(0, z_{2}\right)$ and the unknown parameter $U(0,0)$. This can be done in two steps. First, we notice that $U\left(z_{1}, z_{2}\right)$ must be bounded for all values of $z_{1}$ and $z_{2}$ such that $\left|z_{1}\right| \leq 1$ and $\left|z_{2}\right| \leq 1$. In particular, this should be true for $z_{1}=Y\left(z_{2}\right)$, with $Y\left(z_{2}\right) \triangleq \frac{\left(1-\beta_{1}\right) A\left(Y\left(z_{2}\right), z_{2}\right)}{1-\beta_{1} A\left(Y\left(z_{2}\right), z_{2}\right)}$ and $\left|z_{2}\right| \leq 1$, since it follows by Rouché's theorem that there is exactly one solution for $Y\left(z_{2}\right)$, with $\left|Y\left(z_{2}\right)\right| \leq 1$ for all such $z_{2}$. Notice that $Y(1)$ equals 1 . The above implies that if we choose $z_{1}=Y\left(z_{2}\right)$ in equation (2), where $\left|z_{2}\right| \leq 1$, the denominator of the right hand side of this equation becomes zero. The same must then be true for its numerator, yielding

$$
U\left(0, z_{2}\right)=U(0,0) \frac{Y\left(z_{2}\right)\left(1-\beta_{2}\right)\left(z_{2}-1\right)}{\left(1-\beta_{1}\right) z_{2}-\left(1-\beta_{2}\right) Y\left(z_{2}\right)-\left(\beta_{2}-\beta_{1}\right) Y\left(z_{2}\right) z_{2}}
$$

Finally, in order to find an expression for $U(0,0)$, we put $z_{1}=z_{2}=1$ and use de l'Hopital's rule in equation (2). Therefore we will need to know the value of $Y^{\prime}(1)$. By taking the derivative of both sides of the definition of $Y\left(z_{2}\right)$ and by substituting $z_{2}$ by 1 , we obtain

$$
Y^{\prime}(1)=\frac{\lambda_{2}}{1-\beta_{1}-\lambda_{1}}
$$

Using this expression, we obtain the expected result for $U(0,0)$ :

$$
U(0,0)=1-\rho_{T}
$$

A fully determined expression for $U\left(z_{1}, z_{2}\right)$ can now be derived from equation (2) together with equations (3) and (5):

$$
U\left(z_{1}, z_{2}\right)=\frac{\left(1-\rho_{T}\right)\left(1-\beta_{2}\right) S_{1}\left(A\left(z_{1}, z_{2}\right)\right)\left(z_{2}-1\right)\left(z_{1}-Y\left(z_{2}\right)\right)}{\left(z_{1}-S_{1}\left(A\left(z_{1}, z_{2}\right)\right)\right)\left(\left(1-\beta_{2}\right) Y\left(z_{2}\right)\left(z_{2}-1\right)-\left(1-\beta_{1}\right) z_{2}\left(Y\left(z_{2}\right)-1\right)\right)}
$$

From the two-dimensional pgf $U\left(z_{1}, z_{2}\right)$, we can easily derive an expression for the pgf of the total system contents at the beginning of an arbitrary slot - denoted by $U_{T}(z)$ - yielding

$$
\begin{aligned}
U_{T}(z) & \triangleq \lim _{k \rightarrow \infty} \mathrm{E}\left[z^{u_{T, k}}\right]=U(z, z) \\
& =\frac{\left(1-\rho_{T}\right)\left(1-\beta_{2}\right) S_{1}\left(A_{T}(z)\right)(z-1)(z-Y(z))}{\left(z-S_{1}\left(A_{T}(z)\right)\right)\left(\left(1-\beta_{2}\right) Y(z)(z-1)-\left(1-\beta_{1}\right) z(Y(z)-1)\right)} .
\end{aligned}
$$

We can also derive expressions for the pgf of the steady-state system contents of class 1 and class 2 packets at the beginning of an arbitrary slot - denoted by $U_{1}(z)$ 
and $U_{2}(z)$ respectively - yielding

$$
\begin{aligned}
U_{1}(z) & \triangleq \lim _{k \rightarrow \infty} \mathrm{E}\left[z^{u_{1, k}}\right]=U(z, 1) \\
& =\left(1-\rho_{1}\right) \frac{S_{1}\left(A_{1}(z)\right)(z-1)}{z-S_{1}\left(A_{1}(z)\right)} \\
U_{2}(z) & \triangleq \lim _{k \rightarrow \infty} \mathrm{E}\left[z^{u_{2, k}}\right]=U(1, z) \\
& =\frac{\left(1-\rho_{T}\right)\left(1-\beta_{2}\right) S_{1}\left(A_{2}(z)\right)(z-1)(1-Y(z))}{\left(1-S_{1}\left(A_{2}(z)\right)\right)\left(\left(1-\beta_{2}\right) Y(z)(z-1)-\left(1-\beta_{1}\right) z(Y(z)-1)\right)}
\end{aligned}
$$

We conclude that the system contents of class 1 packets is not influenced by class 2 packets and that its pgf is the same as for a single class system with an identical packet arrival and service process described by $A_{1}(z)$ and $S_{1}(z)$ respectively. This is of course due to the preemptive priority scheduling. For high priority packets, it seems as if no low priority packets are present in the system.

In the special case that $\beta_{j}=0(j=1,2)$, the service times of the packets are deterministic and equal to 1 slot. By substituting $\beta_{1}$ and $\beta_{2}$ by 0 in equation (6), we obtain

$$
U\left(z_{1}, z_{2}\right)=\left(1-\lambda_{T}\right) \frac{A\left(z_{1}, z_{2}\right)\left(z_{2}-1\right)}{z_{1}-A\left(z_{1}, z_{2}\right)} \frac{z_{1}-Y\left(z_{2}\right)}{z_{2}-Y\left(z_{2}\right)}
$$

with $Y(z)=A(Y(z), z)$. This is the same expression as obtained in [20], in which a discrete-time ATM queue with a priority scheduling discipline was analyzed.

\section{Delay}

The packet delay is defined as the total amount of time a packet spends in the system, i.e., the number of slots between the end of the packet's arrival slot and the end of its departure slot. We can analyze the packet delay of class 1 packets as if they are the only packets in the system. This is e.g. done in [1] and the pgf of the packet delay of class 1 packets is given by

$$
D_{1}(z)=\frac{1-\rho_{1}}{\rho_{1}} \frac{z\left(A_{1}\left(S_{1}(z)\right)-1\right)}{z-A_{1}\left(S_{1}(z)\right)} .
$$

The analysis of the packet delay of a class 2 packet is more complicated. The packet delay can be divided into two mutually independent parts. The first part $d_{2}^{(1)}$ is the time a class 2 packet spends in the system from its arrival slot till it enters the server for the first time. The second part of the delay, denoted by $d_{2}^{(2)}$, begins when the tagged packet enters the server for the first time and ends when it leaves the system. So denoting the pgf's of $d_{2}^{(1)}$ and $d_{2}^{(2)}$ by $D_{2}^{(1)}(z)$ and $D_{2}^{(2)}(z)$ respectively, 
the pgf of the delay of a tagged class 2 packet is given by:

$$
D_{2}(z)=D_{2}^{(1)}(z) D_{2}^{(2)}(z)
$$

since $d_{2}^{(1)}$ and $d_{2}^{(2)}$ are independent. The first part is furthermore independent of the fact whether the priority scheduling is preemptive or non-preemptive, i.e., independent of the fact whether newly arriving class 1 packets can interrupt an on-going transmission of a class 2 packet (since $d_{2}^{(1)}$ only accounts for the waiting time in the queue before the tagged class 2 packet enters the server for the first time). The system with a non-preemptive priority scheduling was already analyzed in [21]. We will reconstruct this analysis for the preemptive priority buffer. In order to calculate $D_{2}^{(1)}(z)$, an equivalent virtual system with an altered service discipline is considered. We assume that from the arrival slot of the tagged packet on, denoted by slot $k$, the order of service for class 1 packets (those in the queue at the end of slot $k$ and newly arriving ones) is LCFS instead of FCFS in the equivalent system (the transmission of class 2 packets remains FCFS) and that the priority scheduling is non-preemptive. Let us refer to the packets in the system at the end of the tagged packet's arrival slot, but that have to be served before the tagged packet as the "primary packets". So, a primary packet can enter the server, when the system becomes free (for the first time) of class 1 packets that arrived during and after the service time of the primary packet that predecessed it according to the new service discipline. Let $v_{1, m}^{(i)}$ denote the length of the time period during which the server is occupied by the $m$-th class 1 packet that arrives during slot $i$ and its class 1 "successors", i.e., the time period starting at the beginning of the service of that packet and terminating when the system becomes free (for the first time) of class 1 packets which arrived during and after its service time. Analogously, let $v_{2, m}^{(i)}$ denote the length of the time period during which the server is occupied by the $m$-th class 2 packet that arrives during slot $i$ and its class 1 "successors". The $v_{j, m}^{(i)}$ 's $(j=1,2)$ are called sub-busy periods, caused by the $m$-th class $j$ packet that arrived during slot $i$.

It is clear that the length of the sub-busy periods caused by class 1 packets are i.i.d. and thus have the same pgf $V_{1}(z)$. Also the length of the sub-busy periods caused by class 2 packets are i.i.d., and their pgf is denoted by $V_{2}(z)$. Using the same techniques as in [21], it can then be proven that:

$$
D_{2}^{(1)}(z)=\frac{1-\rho_{T}}{\lambda_{2}} \frac{A\left(V_{1}(z), V_{2}(z)\right)-A_{1}\left(V_{1}(z)\right)}{z A_{1}\left(V_{1}(z)\right)-A\left(V_{1}(z), V_{2}(z)\right)}\left(1-\beta_{2} z A_{1}\left(V_{1}(z)\right)\right)
$$


with $V_{j}(z)=\frac{\left(1-\beta_{j}\right) z A_{1}\left(V_{1}(z)\right)}{1-\beta_{j} z A_{1}\left(V_{1}(z)\right)}(j=1,2)$, and where we have explicitly used the expressions of the pgf's of the geometrical service times.

The second part of the delay of the tagged class 2 packet begins when the tagged packet enters the server for the first time. If class 1 packets arrive before the service of the tagged packet is finished, the packet can not be fully transmitted and it has to wait to continue its service until all class 1 packets have left the system. So, each class 1 packet that arrives during the service of the tagged packet, starts a sub-busy period. Taking this into account, the following expression for $D_{2}^{(2)}(z)$ can be found:

$$
D_{2}^{(2)}(z)=\frac{\left(1-\beta_{2}\right) z}{1-\beta_{2} z A_{1}\left(V_{1}(z)\right)}
$$

Finally, using (13) and (14) in equation (12), we find:

$$
D_{2}(z)=\frac{1-\rho_{T}}{\rho_{2}} \frac{z\left(A\left(V_{1}(z), V_{2}(z)\right)-A_{1}\left(V_{1}(z)\right)\right)}{z A_{1}\left(V_{1}(z)\right)-A\left(V_{1}(z), V_{2}(z)\right)}
$$

with $V_{j}(z)=\frac{\left(1-\beta_{j}\right) z A_{1}\left(V_{1}(z)\right)}{1-\beta_{j} z A_{1}\left(V_{1}(z)\right)}(j=1,2)$.

\section{Calculation of moments}

The functions $Y(z), V_{1}(z)$ and $V_{2}(z)$ can only be explicitly found in case of some simple arrival processes. Their derivatives, necessary to calculate the moments of the system contents and the packet delay, on the contrary, can be calculated in closed-form. For example, $Y^{\prime}(1)$ is given by equation (4) and the first derivatives of $V_{j}(z)$ for $z=1$ are given by

$$
V_{j}^{\prime}(1)=\frac{1}{1-\beta_{j}-\lambda_{1}}
$$

with $j=1,2$. Let us define $\lambda_{i j}$ as

$$
\left.\lambda_{i j} \triangleq \frac{\partial^{2} A\left(z_{1}, z_{2}\right)}{\partial z_{i} \partial z_{j}}\right|_{z_{1}=z_{2}=1},
$$

with $i, j=1,2$. Now we can calculate the mean total system contents, the mean system contents of class 1 and class 2 packets and the mean packet delay of both classes by taking the first derivatives of the respective pgfs for $z=1$. We find

$$
E\left[u_{T}\right]=\lambda_{T}+\frac{\rho_{T}\left(1-\rho_{1}\right)-\rho_{1} \rho_{2}-\lambda_{1}\left(1-\rho_{T}\right)}{\left(1-\rho_{T}\right)\left(1-\rho_{1}\right)}-\frac{\lambda_{2}\left(1-\beta_{1}-\rho_{1}\right)}{\left(1-\beta_{1}\right)\left(1-\rho_{T}\right)\left(1-\rho_{1}\right)}
$$




$$
+\frac{\left(\left(1-\beta_{1}\right)\left(1-\rho_{T}\right)+\lambda_{2}\right) \lambda_{11}}{2\left(1-\beta_{1}\right)^{2}\left(1-\rho_{T}\right)\left(1-\rho_{1}\right)}+\frac{\lambda_{12}}{\left(1-\beta_{1}\right)\left(1-\rho_{T}\right)}+\frac{\lambda_{22}}{2\left(1-\beta_{2}\right)\left(1-\rho_{T}\right)},
$$

for the mean total system contents,

$$
E\left[u_{1}\right]=\frac{\left(1-\lambda_{1}\right) \rho_{1}}{1-\rho_{1}}+\frac{1}{2} \frac{\lambda_{11}}{\left(1-\beta_{1}\right)\left(1-\rho_{1}\right)},
$$

for the mean system contents of class 1 packets and

$$
\begin{aligned}
E\left[u_{2}\right]= & \lambda_{2}+\frac{\rho_{2}}{1-\rho_{T}}-\frac{\lambda_{2}\left(1-\beta_{1}-\rho_{1}\right)}{\left(1-\beta_{1}\right)\left(1-\rho_{T}\right)\left(1-\rho_{1}\right)}+\frac{\lambda_{22}}{2\left(1-\beta_{2}\right)\left(1-\rho_{T}\right)} \\
& +\frac{\lambda_{12}}{\left(1-\beta_{1}\right)\left(1-\rho_{T}\right)}+\frac{\lambda_{2} \lambda_{11}}{2\left(1-\beta_{1}\right)^{2}\left(1-\rho_{T}\right)\left(1-\rho_{1}\right)}
\end{aligned}
$$

for the mean system contents of class 2 packets. It is easily verified that equations (16) - (18) satisfy $E\left[u_{T}\right]=E\left[u_{1}\right]+E\left[u_{2}\right]$. Furthermore, we find

$$
E\left[d_{1}\right]=\frac{1}{1-\beta_{1}}+\frac{\beta_{1} \rho_{1}}{\left(1-\beta_{1}\right)\left(1-\rho_{1}\right)}+\frac{\lambda_{11}}{2 \lambda_{1}\left(1-\beta_{1}\right)\left(1-\rho_{1}\right)}
$$

for the mean packet delay of a class 1 packet and

$$
\begin{aligned}
E\left[d_{2}\right]= & 1+\frac{1}{\left(1-\beta_{2}\right)\left(1-\rho_{T}\right)}+\frac{\lambda_{22}}{2 \lambda_{2}\left(1-\beta_{2}\right)\left(1-\rho_{T}\right)}+\frac{\lambda_{12}}{\lambda_{2}\left(1-\beta_{1}\right)\left(1-\rho_{T}\right)} \\
& -\frac{\left(1-\beta_{1}-\rho_{1}\right)}{\left(1-\beta_{1}\right)\left(1-\rho_{T}\right)\left(1-\rho_{1}\right)}+\frac{\lambda_{11}}{2\left(1-\beta_{1}\right)^{2}\left(1-\rho_{T}\right)\left(1-\rho_{1}\right)}
\end{aligned}
$$

for the mean packet delay of a class 2 packet. Note that equations (17) - (20) satisfy Little's law $E\left[d_{j}\right]=E\left[u_{j}\right] / \lambda_{j}$.

In a similar way, expressions for the variance (and higher moments) of the random variables of interest can be calculated by taking the appropriate derivatives of the respective generating functions as well. These expressions are however too elaborate to show, but figures of variances of system contents and packet delay of both classes will be shown in section 7 .

\section{Tail behavior}

Not only the moments of the system contents and cell delay are important performance measures, but also, and especially, the tail distribution of these quantities, which are often used to impose statistical bounds on the guaranteed QoS for both classes.

From the generating functions of the total system contents, and of the system contents and cell delay of class 1 and class 2 cells derived in sections 3 and 4, approx- 
imations of the tail probabilities can be derived using complex contour integration and residue theory. The procedure to find the corresponding probability mass function of a pgf, frequently used in the remainder of this section, is described in general terms in Appendix 1.

In order to determine the asymptotic behavior of the tail distribution, the dominant singularity of the respective generating functions is important. In e.g. [2] (wherein a single-class ATM queue with a FIFO scheduling discipline is analyzed), it is proven that the dominant singularity lies on the positive real axis and is larger than 1.

First we concentrate on the system contents of class 1. Provided that the pgf's $A_{1}(z)$ and $S_{1}(z)$ exhibit no long-tail behavior, which is assumed to be the case here, the dominant singularity $z_{H}$ of $U_{1}(z)$ is a zero of $z-S_{1}\left(A_{1}(z)\right)$ and this singularity is a single pole. In the neighbourhood of this pole, we can approximate $U_{1}(z)$ by

$$
U_{1}(z) \approx \frac{K_{1}}{z_{H}-z}
$$

where $K_{1}$ can be found by substituting $z=z_{1}$ in (21). Using residue theory, the tail probability is easily found to yield

$$
\operatorname{Prob}\left[u_{1}=n\right] \approx\left(1-\rho_{1}\right) \frac{z_{H}-1}{S_{1}^{\prime}\left(A_{1}\left(z_{H}\right)\right) A_{1}^{\prime}\left(z_{H}\right)-1} z_{H}^{-n}
$$

for large enough $n$.

The tail behavior of the system contents of class 2 packets is a bit more involved, since it is not a priori clear what the dominant singularity of $U_{2}(z)$ is. This is due to the occurance of the function $Y(z)$ in (9), which is only implicitly defined. First we take a closer look at that function $Y(z)$. The first derivative of $Y(z)$ is given by

$$
Y^{\prime}(z)=\frac{\left(1-\beta_{1}\right) A^{(2)}(Y(z), z)}{\left(1-\beta_{1} A(Y(z), z)\right)^{2}-\left(1-\beta_{1}\right) A^{(1)}(Y(z), z)},
$$

with $\left.A^{(j)}(y, z) \triangleq \frac{\partial A\left(z_{1}, z_{2}\right)}{\partial z_{j}}\right|_{z_{1}=y, z_{2}=z}(j=1,2)$. Consequently, $Y(z)$ has a singularity, denoted as $z_{B}$, where the denominator of $Y^{\prime}(z)$ becomes 0 , i.e., $(1-$ $\left.\beta_{1} A\left(Y\left(z_{B}\right), z_{B}\right)\right)^{2}=\left(1-\beta_{1}\right) A^{(1)}\left(Y\left(z_{B}\right), z_{B}\right)$. Since $Y(z)$ remains finite in the neighborhood of $z_{B}$, this singularity is not a simple pole. Applying the results from [4] one can show that in the neighbourhood of $z_{B}, Y(z)$ is approximately given by

$$
Y(z) \approx Y\left(z_{B}\right)-K_{Y} \sqrt{z_{B}-z}
$$


with

$$
K_{Y}=\sqrt{\frac{2\left(1-\beta_{1}\right) A^{(2)}\left(Y\left(z_{B}\right), z_{B}\right)}{2 \beta_{1}\left(1-\beta_{1} A\left(Y\left(z_{B}\right), z_{B}\right)\right) A^{(1)}\left(Y\left(z_{B}\right), z_{B}\right)+\left(1-\beta_{1}\right) A^{(11)}\left(Y\left(z_{B}\right), z_{B}\right)}},
$$

which can be found by taking the limit $z \rightarrow z_{B}$ of $(24) . A^{(i j)}(y, z)$ is defined as $\left.\frac{\partial^{2} A\left(z_{1}, z_{2}\right)}{\partial z_{i} \partial z_{j}}\right|_{z_{1}=y, z_{2}=z}$ (for $\left.i, j=1,2\right)$. From equation (24) it becomes obvious that $z_{B}$ is a square-root branch point of $Y(z)$. $Y(z)$ has thus two real solutions when $z<z_{B}$ (the solution we are interested in is the one where $Y(z)<1$, if $z<1$ ), which coincide at $z_{B}$, and has no real solution when $z>z_{B}$. $z_{B}$ is then of course also a branch point of $U_{2}(z)$. A second potential singularity $z_{L}$ of $U_{2}(z)$ on the real axis is given by the positive zero of the denominator which is a zero of $\left(1-\beta_{2}\right) Y(z)(z-1)-\left(1-\beta_{1}\right) z(Y(z)-1)$.

The tail behavior of the system contents of class 2 packets is thus characterized by $z_{L}$ or $z_{B}$, depending on which is the dominant (i.e., smallest) singularity. Three types of tail behavior may thus occur, namely when $z_{L}<z_{B}, z_{L}=z_{B}$ and $z_{L}$ does not exist. In those three cases, $U_{2}(z)$ can be approximated in the neighbourhood of its dominant singularity by:

$$
U_{2}(z) \approx \begin{cases}\frac{K_{2}^{(1)}}{z_{L}-z} & \text { if } z_{L}<z_{B} \\ \frac{K_{2}^{(2)}}{\sqrt{z_{B}-z}} & \text { if } z_{L}=z_{B} \\ U_{2}\left(z_{B}\right)-K_{2}^{(3)} \sqrt{z_{B}-z} & \text { if } z_{L} \text { does not exist }\end{cases}
$$

where the constants $K_{2}^{(i)}(i=1,2,3)$ can be found by investigating the behavior of $U_{2}(z)$ in the neighbourhood of this dominant singularity. Using residue theory, we find the tail probabilities for the three possible cases:

$$
\operatorname{Prob}\left[u_{2}=n\right] \approx\left\{\begin{array}{l}
\left(1-\rho_{T}\right) \frac{\left(1-\beta_{2}\right) S_{1}\left(A_{2}\left(z_{L}\right)\right)\left(z_{L}-1\right)\left(1-Y\left(z_{L}\right)\right) z_{L}^{-n}}{z_{L}\left(S_{1}\left(A_{2}\left(z_{L}\right)\right)-1\right) Q_{1}\left(z_{L}\right)} \\
\frac{1-\rho_{T}}{K_{Y}} \sqrt{\frac{1}{z_{B} \pi}} \frac{\left(1-\beta_{2}\right) S_{1}\left(A_{2}\left(z_{B}\right)\right)\left(z_{B}-1\right)\left(1-Y\left(z_{B}\right)\right) n^{-1 / 2} z_{B}^{-n}}{\left(S_{1}\left(A_{2}\left(z_{B}\right)\right)-1\right)\left(\left(1-\beta_{2}\right)\left(z_{B}-1\right)-\left(1-\beta_{1}\right) z_{B}\right)} \\
\frac{\left(1-\rho_{T}\right) K_{Y}}{2} \sqrt{\frac{z_{B}}{\pi}} \frac{\left(1-\beta_{2}\right)^{2} S_{1}\left(A_{2}\left(z_{B}\right)\right)\left(z_{B}-1\right)^{2} n^{-3 / 2} z_{B}^{-n}}{\left(S_{1}\left(A_{2}\left(z_{B}\right)\right)-1\right)\left(Q_{2}\left(z_{B}\right)\right)^{2}}
\end{array}\right.
$$

for large enough $n$, if $z_{L}<z_{B}$, if $z_{L}=z_{B}$, and if $z_{L}$ does not exist respectively. The functions $Q_{j}(z)(j=1,2)$ are defined as

$$
Q_{1}(z)=\left(1-\beta_{2}\right)\left(Y^{\prime}(z)(z-1)+Y(z)\right)-\left(1-\beta_{1}\right)\left(Y(z)-1+z Y^{\prime}(z)\right)
$$




$$
Q_{2}(z)=\left(1-\beta_{2}\right) Y(z)(z-1)-\left(1-\beta_{1}\right) z(Y(z)-1)
$$

respectively. The first expression of (25) (as well as expression (22)) constitutes a typical geometric tail behavior (i.e., an exponantial decay), the third expression is a typical non-geometric tail behavior, while the second expression gives a transition between geometric and non-geometric tail behavior. Equation (25) is found from the approximations of the generating functions by applying the Theorem from Appendix 2 (which is a theorem stated in [6]).

The tail behavior of the total system contents is similar to the tail of the low priority system contents. The important singularities of $U_{T}(z)$ are the same as for $U_{2}(z)$, i.e., $z_{L}$ and $z_{B}$. So, $U_{T}(z)$ can be approximated in the neighbourhood of its dominant singularity by:

$$
U_{T}(z) \approx \begin{cases}\frac{K_{T}^{(1)}}{z_{L}-z} & \text { if } z_{L}<z_{B} \\ \frac{K_{T}^{(2)}}{\sqrt{z_{B}-z}} & \text { if } z_{L}=z_{B} \\ U_{T}\left(z_{B}\right)-K_{T}^{(3)} \sqrt{z_{B}-z} & \text { if } z_{L} \text { does not exist }\end{cases}
$$

where the constants $K_{T}^{(i)}(i=1,2,3)$ can again be found by investigating the behavior of $U_{T}(z)$ in the neighbourhood of this dominant singularity. Using residue theory, we find the tail probabilities for the three cases:

$$
\operatorname{Prob}\left[u_{T}=n\right] \approx\left\{\begin{array}{l}
\left(1-\rho_{T}\right) \frac{\left(1-\beta_{2}\right) S_{1}\left(A_{T}\left(z_{L}\right)\right)\left(z_{L}-1\right)\left(z_{L}-Y\left(z_{L}\right)\right) z_{L}^{-n}}{z_{L}\left(S_{1}\left(A_{T}\left(z_{L}\right)\right)-z_{L}\right) Q_{1}\left(z_{L}\right)} \\
\frac{1-\rho_{T}}{K_{Y} \sqrt{z_{B} \pi}} \frac{\left(1-\beta_{2}\right) S_{1}\left(A_{T}\left(z_{B}\right)\right)\left(z_{B}-1\right)\left(z_{B}-Y\left(z_{B}\right)\right) n^{-1 / 2} z_{B}^{-n}}{\left(S_{1}\left(A_{T}\left(z_{B}\right)\right)-z_{B}\right)\left(\left(1-\beta_{2}\right)\left(z_{B}-1\right)-\left(1-\beta_{1}\right) z_{B}\right)} \\
\frac{\left(1-\rho_{T}\right) K_{Y}}{2 \sqrt{\pi / z_{B}}} \frac{\left(1-\beta_{2}\right)\left(\beta_{2}-\beta_{1}\right) S_{1}\left(A_{T}\left(z_{B}\right)\right)\left(z_{B}-1\right)^{2} n^{-3 / 2} z_{B}^{-n+1}}{\left(z_{B}-S_{1}\left(A_{T}\left(z_{B}\right)\right)\right)\left(Q_{2}\left(z_{B}\right)\right)^{2}}
\end{array}\right.
$$

for large enough $n$, if $z_{L}<z_{B}$, if $z_{L}=z_{B}$, and if $z_{L}$ does not exist respectively.

Let us now consider the packet delay. The dominant singularity of $D_{1}(z)$ is a zero of $z-A_{1}\left(S_{1}(z)\right)$, denoted by $\hat{z}_{H}$, and we can thus approximate the tail behavior of the delay of class 1 packets by

$$
\operatorname{Prob}\left[d_{1}=n\right] \approx \frac{\left(1-\rho_{1}\right)}{\rho_{1}} \frac{\hat{z}_{H}-1}{A_{1}^{\prime}\left(S_{1}\left(\hat{z}_{H}\right)\right) S_{1}^{\prime}\left(\hat{z}_{H}\right)-1} z_{H}^{-n}
$$

for large enough $n$. The calculation of these tail probabilities is similar to the calculation of (22). The tail behavior of the delay of class 2 packets is again a bit 
more involved because of the appearance of the function $V_{1}(z)$ (and $V_{2}(z)$ ) in (15), which is only implicitly known. The first derivative of $V_{1}(z)$ is given by

$$
V_{1}^{\prime}(z)=\frac{\left(1-\beta_{1}\right) A_{1}\left(V_{1}(z)\right)}{\left(1-\beta_{1} z A_{1}\left(V_{1}(z)\right)\right)^{2}-\left(1-\beta_{1}\right) z A_{1}^{\prime}\left(V_{1}(z)\right)},
$$

which, similar as before, indicates that $V_{1}(z)$ also has a square root branch point $\hat{z}_{B}$, with $\left(1-\beta_{1} \hat{z}_{B} A_{1}\left(V_{1}\left(\hat{z}_{B}\right)\right)\right)^{2}=\left(1-\beta_{1}\right) \hat{z}_{B} A_{1}^{\prime}\left(V_{1}\left(\hat{z}_{B}\right)\right)$. In the neighbourhood of $\hat{z}_{B}, V_{1}(z)$ is approximately given by

$$
V_{1}(z) \approx V_{1}\left(\hat{z}_{B}\right)-K_{V} \sqrt{\hat{z}_{B}-z}
$$

with

$$
K_{V}=\sqrt{\frac{2\left(1-\beta_{1}\right) A_{1}\left(V\left(\hat{z}_{B}\right)\right)}{\hat{z}_{B}\left[2 \beta_{1}\left(1-\beta_{1} \hat{z}_{B} A_{1}\left(V_{1}\left(\hat{z}_{B}\right)\right)\right) A_{1}^{\prime}\left(V_{1}\left(\hat{z}_{B}\right)\right)+\left(1-\beta_{1}\right) A_{1}^{\prime \prime}\left(V\left(\hat{z}_{B}\right)\right)\right]}} .
$$

A second singularity of $D_{2}(z)$ is given by the dominant zero $\hat{z}_{L}$ of $z A_{1}\left(V_{1}(z)\right)-$ $A\left(V_{1}(z), V_{2}(z)\right)$ on the real positive axis.

So, $D_{2}(z)$ can be approximated in the neighbourhood of his dominant singularity by:

$$
D_{2}(z) \approx \begin{cases}\frac{\hat{K}_{2}^{(1)}}{\hat{z}_{L}-z} & \text { if } \hat{z}_{L}<\hat{z}_{B} \\ \frac{\hat{K}_{2}^{(2)}}{\sqrt{\hat{z}_{B}-z}} & \text { if } \hat{z}_{L}=\hat{z}_{B} \\ D_{2}\left(\hat{z}_{B}\right)-\hat{K}_{2}^{(3)} \sqrt{\hat{z}_{B}-z} & \text { if } \hat{z}_{L} \text { does not exist }\end{cases}
$$

where the constants $\hat{K}_{2}^{(i)}(i=1,2,3)$ can be found by investigating $D_{2}(z)$ in the neighbourhood of its dominant singularity. By using residue theory once again, the asymptotic behavior of $D_{2}(z)$ is given by

$$
\operatorname{Prob}\left[d_{2}=n\right] \approx\left\{\begin{array}{l}
\frac{\left(1-\rho_{T}\right) A_{1}\left(V_{1}\left(\hat{z}_{L}\right)\right)\left(\hat{z}_{L}-1\right) \hat{z}_{L}^{-n} / \rho_{2}}{\left.\frac{d A\left(V_{1}(z), V_{2}(z)\right)}{d z}\right|_{z=\hat{z}_{L}}-A_{1}\left(V_{1}\left(\hat{z}_{L}\right)\right)-\hat{z}_{L} A_{1}^{\prime}\left(V_{1}\left(\hat{z}_{L}\right)\right) V_{1}^{\prime}\left(\hat{z}_{L}\right)} \\
\frac{\left(1-\rho_{T}\right)\left(\hat{z}_{B}-1\right) A_{1}\left(V_{1}\left(\hat{z}_{B}\right)\right)\left(1-\beta_{2} \hat{z}_{B} A_{1}\left(V_{1}\left(\hat{z}_{B}\right)\right)\right)^{2} n^{-1 / 2} \hat{z}_{B}^{-n}}{\rho_{2} K_{V} \sqrt{\pi / \hat{z}_{B}}\left(Q_{3}\left(\hat{z}_{B}\right)-\hat{z}_{B} A_{1}^{\prime}\left(V_{1}\left(\hat{z}_{B}\right)\right)\right)\left(1-\beta_{2} \hat{z}_{B} A_{1}\left(V_{1}\left(\hat{z}_{B}\right)\right)\right)^{2}} \\
\frac{\left(1-\rho_{T}\right) K_{V}}{2 \rho_{2} \sqrt{\pi / \hat{z}_{B}}} \frac{\left.\hat{z}_{B}\left(\hat{z}_{B}-1\right) Q_{4}\left(\hat{z}_{B}\right) n^{-3 / 2} \hat{z}_{B}^{-n}\left(V_{1}\left(\hat{z}_{B}\right)\right)-A\left(V_{1}\left(\hat{z}_{B}\right), V_{2}\left(\hat{z}_{B}\right)\right)\right)^{2}}{(3},
\end{array}\right.
$$

if $\hat{z}_{L}<\hat{z}_{B}$, if $\hat{z}_{L}=\hat{z}_{B}$, and if $\hat{z}_{L}$ does not exist respectively. $Q_{j}(z)(j=3,4)$ are 
defined as follows:

$$
\begin{aligned}
& Q_{3}(z)=A^{(1)}\left(V_{1}(z), V_{2}(z)\right)+\frac{\left(1-\beta_{2}\right) z A_{1}^{\prime}\left(V_{1}(z)\right) A^{(2)}\left(V_{1}(z), V_{2}(z)\right)}{\left(1-\beta_{2} z A_{1}\left(V_{1}(z)\right)\right)^{2}} \\
& Q_{4}(z)=A_{1}\left(V_{1}(z)\right) Q_{3}(z)-A\left(V_{1}(z), V_{2}(z)\right) A_{1}^{\prime}\left(V_{1}(z)\right)
\end{aligned}
$$

The first expression of (30) has a typical geometric tail behavior, the third expression has a typical non-geometric tail behavior and the second expression gives a transition between the two former situations.

A quantity of practical interest is the probability that a packet has a delay that exceeds a bound $D$. We find

$$
\operatorname{Prob}\left[d_{1}>D\right] \approx \frac{\operatorname{Prob}\left[d_{1}=D+1\right] \hat{z}_{H}}{\hat{z}_{H}-1}
$$

for the probability that the delay of a class 1 packet is larger than a bound $D$. This can be found by summing equation (27) for all appropriate values of $n$. Analogously, we can calculate the probability that a class 2 packet exceeds a bound $\mathrm{D}$ by summing equation (30) for the appropriate values of $n$. We find

$$
\operatorname{Prob}\left[d_{2}>D\right] \approx \begin{cases}\frac{\operatorname{Prob}\left[d_{2}=D+1\right] \hat{z}_{L}}{\hat{z}_{L}-1} & \text { if } \hat{z}_{L}<\hat{z}_{B} \\ \frac{\operatorname{Prob}\left[d_{2}=D+1\right] \hat{z}_{B}}{\hat{z}_{B}-1} & \text { if } \hat{z}_{L}=\hat{z}_{B} \\ \frac{\operatorname{Prob}\left[d_{2}=D+1\right] \hat{z}_{B}}{\hat{z}_{B}-1} & \text { if } \hat{z}_{L} \text { does not exist }\end{cases}
$$

where we used the approximation that $\sum_{n=D+1}^{\infty} n^{-a} z^{-n} \approx(D+1)^{-a} \sum_{n=D+1}^{\infty} z^{-n}$, with $a=1 / 2$ or $3 / 2$ and which holds for large enough $D$. Some similar expressions can be found for the probability that the system contents exceeds a certain bound.

Since the results obtained in this section are approximate (due to the dominant pole approximative method), the question remains if the expressions are accurate. From the analysis in [2], it follows that the approximation of the tail probabilities, obtained through the dominant pole method, are better when the dominant pole is more dominant (i.e., the higher the moduli of the other poles compared to the modulus of the dominant pole, the better the quality of the approximation) and for higher values of the random variable under study (i.e., coefficient $n$ in expressions (22), (25)-(27) and (30) is higher). We will show in section 7 that the approximate results for the tail probabilities obtained in this section are more than satisfactory. 


\section{Numerical examples}

\subsection{An $N \mathbf{x} N$ switch}

In this subsection, we present some numerical examples. We assume the traffic of the two classes to be arriving according to a two-dimensional binomial process. Its two-dimensional pgf is given by:

$$
A\left(z_{1}, z_{2}\right)=\left(1-\frac{\lambda_{1}}{N}\left(1-z_{1}\right)-\frac{\lambda_{2}}{N}\left(1-z_{2}\right)\right)^{N}
$$

The arrival rate of class $j$ traffic is thus given by $\lambda_{j}(j=1,2)$. This arrival process occurs for instance at an output queue of an $N \mathrm{x} N$ output queueing switch fed by a Bernoulli process at the inlets (see [20]). Notice also that if $N \rightarrow \infty$, the arrival process is a superposition of two independent Poisson streams. In the remainder of this section, we assume that $N=16$.

In Figures 1 and 2, the mean and variance of the system contents of class 1 and class 2 packets is shown as a function of the total load $\rho_{T}$, when $\beta_{1}=\beta_{2}=0.5$ (i.e., $\mu_{1}=\mu_{2}=2$ ). The fraction of the class 1 load in the total load, denoted by $\alpha$, is $0.25,0.5$ and 0.75 respectively. We clearly see the influence of the priority scheduling. The mean and variance of the system contents of class 1 packets remains low, even if the fraction of class 1 packets is high. The mean and variance of the system contents of class 2 packets on the other hand is large.

[Figure 1 about here.]

[Figure 2 about here.]

In Figures 3 and 4, the mean value and variance of the packet delay of class 1 and class 2 packets is shown as a function of the total load $\rho_{T}$, when again $\beta_{1}=\beta_{2}=0.5$ and $\alpha=0.25,0.5$ and 0.75 respectively. In order to compare with FIFO scheduling, we have also shown the mean value and variance of the packet delay in that case. Since, in this example, the service times of the class 1 and class 2 packets are equal, the packet delay is the same for class 1 and class 2 packets, and can thus be calculated as if there is only one class of packets arriving according to an arrival process with $\operatorname{pgf} A_{T}(z)$. This has already been analyzed, e.g., in [1]. The influence of priority scheduling on the packet delay becomes obvious from these figures: mean and variance of the delay of class 1 packets reduces significantly. The price to pay is of course a larger mean and variance of the delay of class 2 packets. If this kind of traffic is not delay-sensitive, as assumed, this is not a too big a problem. Also, 
the smaller the fraction of high priority packets in the overall traffic mix, the lower the mean and variance of the packet delay of both classes will be.

[Figure 3 about here.]

[Figure 4 about here.]

Figure 5 (Figure 6 respectively) shows the mean delay of high and low priority packets as a function of the mean service time of the low priority packets (high priority packets respectively), i.e., $\mu_{2}$ ( $\mu_{1}$ respectively), when $\mu_{1}=2\left(\mu_{2}=2\right.$ respectively), $\rho_{T}=0.75$ and $\alpha=0.25,0.5$ and 0.75 . The figures show that the mean packet delay of high priority packets is not influenced by the mean service time of class 2 packets, while it is proportionally increasing with the mean service time of class 1 packets (when the load of high and low priority packets is kept constant). The mean packet delay of class 2 packets on the other hand is proportionally increasing with the mean service time of class 2 packets (Figure 5) and with the mean service time of class 1 packets (Figure 6). Because of the preemptive priority scheduling, mean delay of high priority packets is only influenced by its own arrival and service process, while the mean delay of low priority packets is influenced by the arrival and service processes of both classes.

[Figure 5 about here.]

[Figure 6 about here.]

In Figure 7, the mean delay of both classes is shown as a function of the number of in- and outlets $N$, for $\beta_{j}=0.5(j=1,2)$. We have also plotted the mean delay of the low-priority packets when the correlation between the number of arrivals of both classes is neglected (in which case the joint pgf of the number of packet arrivals of both classes is set equal to the product of their respective marginal pgf's). The figure shows that the mean delay of both classes grows with the number of inlets for approximately $N<16$ and stays constant for higher $N$ since, for high enough values of $N$, the joint arrival process evolves towards two independent Poisson processes, as explained before. It also shows that if the correlation between the number of arrivals of both classes was neglected there would be an overestimation of the mean low priority delay for small $N$. For higher number of inlets, this correlation factor is neglegible. Since $\mathrm{E}\left[d_{1}\right]$ does not depend on the arrival process of class 2 packets, the curves for the mean high priority packet delay are the same with or without the correlation. 
[Figure 7 about here.]

In the next figures, we illustrate the tail behavior of the packet delay distribution. The tail behavior of the system contents distribution is similar (see section 6) and will not be shown here. We have shown in section 6 that the tails of the class 2 packet delay distribution can have 3 types of asymptotic behavior, depending on which singularity of the respective pgf's is dominant. In case of the output queueing switch considered in this section, Figure 8 shows for which combination of class 1 and class 2 loads the transition type behavior occurs for the class 2 packet delay, i.e., for which combination of arrival rates the regular pole and the branch point coincide, when $\beta_{1}=0.5$ and for varying values of $\beta_{2}$. In the region above the curves, the class 2 tail behavior is geometric for the respective $\rho_{1}$ and $\rho_{2}$, while below the curves the class 2 tail behavior is typically non-geometric. Note that in the area above the line defined by $\rho_{1}+\rho_{2}=1$ in Figure 8 , the total load is larger than 1 , and as a result, the system becomes unstable. As can be seen, the higher $\beta_{2}$ the smaller the region where the class 2 tail behavior is non-geometric.

[Figure 8 about here.]

Figure 9 shows the tail behavior of the packet delay of class 1 and class 2 packets with $\lambda_{1}=0.2, \beta_{1}=\beta_{2}=0.5$, and $\lambda_{2}=0.05$ (non-geometric behavior), approximately 0.116 (transition type behavior) and 0.2 (geometric behavior) respectively. Tail behavior of packet delay of class 1 packets is of course the same for the three cases, since the arrival process of class 1 packets is identical and class 2 packets are 'invisible' for the high priority packets. We have also compared our approximations with simulation results (marks in the figures). The figures show that the approximations for the class 1 , the geometric and transition type tail behavior of class 2 delay is very good in these cases. The approximations of the tails for the non-geometric case are not as good, but still more than satisfactory (for large enough $n$ ).

[Figure 9 about here.]

To conclude this section, we analyse the following case-study. Consider two traffic classes generating packets that arrive in a common multiplexer buffer where they are temporarily stored before transmission. The packet arrival process of both classes is described by a joint pgf given by expression (32). The mean service time of both classes is equal to 2 . For both classes, their respective packet delay must satisfy the constraint $\operatorname{Prob}\left[d_{j}>T_{j}\right]<10^{-X_{j}}$, i.e., the fraction of packets of class$j$ that have a delay larger than the treshold $T_{j}$ may not exceed $10^{-X_{j}}$, where $T_{j}$ 
and $X_{j}$ depend on the application under consideration. It is assumed that class 1 packets are delay-sensitive, implying that they are given priority over class 2 packets (and $T_{1}<T_{2}$, since it makes no sense to have a higher delay treshold for delaysensitive traffic). Class 2 traffic may be loss-sensitive, and the amount of packets that is rejected due to a delay treshold being exceeded must be sufficiently small. Therefore, in the remainder we will set $X_{2}=9$ and $X_{1} \equiv X$ (where the latter may be varied). It is clear that the performance of both traffic classes, in particular their delay characteristics, can be studied using the results derived throughout this paper.

The question we wish to answer is the following: what is the maximal load (denoted by $\rho_{T, \max }$ ), as a function of the traffic mix $\alpha$, that still fulfils the two constraints? In Figure 10, we show the maximal load as a funtion of $\alpha$ when $T_{1}=20$, $T_{2}=100$ and several values of $X$. The constraint for the delay of class 2 packets is the same for all $X$, i.e., $\operatorname{Prob}\left[d_{2}>100\right]<10^{-9}$. For $X<5$, we see that this constraint is the decisive one. We notice that the maximal load suddenly lowers a reasonable amount when $\alpha$ reaches approximately 0.45 . At this point, the tail behavior changes from geometric to non-geometric tail behavior. The sudden change near 0.45 is probably due to the lack of accurateness in the tail behavior of the class 2 delay near the transition. Near this value for $\alpha$, the maximal load we find is thus not that accurate, but one can see that the incorrectness is in the order of a few percentages. For higher $X(X=5)$, the constraint for the delay of the high-priority traffic becomes decisive for high $\alpha$, i.e. when more class 1 packets arrive. For even higher $X$, the class 1 constraint can only be fulfilled for very low class 1 loads. The reason for this is that $\operatorname{Prob}\left[s_{1}>20\right]$ (with $s_{1}$ the service time of a class 1 packet) is very close to $10^{-X}$. Since the service time of a class 1 packet is part of its delay, $\operatorname{Prob}\left[d_{1}>20\right]<10^{-X}$ can only be fulfilled when there's nearly no queueing for class 1 packets, i.e., for low $\alpha$ and low $\rho_{T}$. In Figure 11, we show $\rho_{T, \max }$ as a funtion of $\alpha$ when $X=4, T_{2}=100$ and $T_{1} \geq 14$. The constraint for the delay of class 2 packets is again the same for all $T_{1}$. For $T_{1}>17$, we see that this constraint is the decisive one. For lower $T_{1}$, the constraint for the delay of the high priority traffic becomes decisive for high $\alpha$, i.e. when more class 1 packets arrive. Finally, in Figure 12, the maximum load as a function of $\alpha$ is shown, when $X=4, T_{1}=17$ and several values of $T_{2}$. For low $T_{2}$, the constraint for the low priority traffic is always the most stringent, while for $T_{2} \geq 100$, the constraint for the high priority traffic is decisive for high $\alpha$.

The behavior depicted in these three figures can be explained as follows. For $\alpha=0$, the traffic mix consists of low priority packets only, and $\rho_{T, \max }$ is relatively 
high, depending on the value of $T_{2}$. As $\alpha$ increases, $\rho_{T, \max }$ gradually decreases (but is still determined by $T_{2}$ ) since the growing fraction of high priority packets causes the mean low priority packet delay to rise. Then, as $\alpha$ further increases, a transition point is reached, which is defined as the value of $\alpha$ and $\rho_{T}$ for which $\operatorname{Prob}\left[d_{1}>T_{1}\right]=10^{-X}$ and $\operatorname{Prob}\left[d_{2}>T_{2}\right]=10^{-9}$. Beyond this transition point, the bounding set by $T_{1}$ becomes predominant, and $\rho_{T, \max }$ further decreases due to the ever increasing presence of high priority packets in the traffic mix. These figures show that the maximum allowable load can strongly depend on the delay boundaries $T_{1}$ and $T_{2}$ set on the high and low priority packet delays, and the traffic mix $\alpha$.

[Figure 10 about here.]

[Figure 11 about here.]

[Figure 12 about here.]

\subsection{Impact of the correlation between both classes}

Figure 7 shows the impact of neglecting the correlation in an $N \mathrm{x} N$ switch. In this subsection, we will further study the impact of the correlationfactor of the number of per-slot arrivals of both classes on the mean delay of class 2 packets. Therefore, we assume a simple arrival process as follows: the marginal distribution of the class $j$ packets are Bernoulli distributed and thus given by: $\operatorname{Prob}\left[a_{j, k}=0\right]=1-\lambda_{j}$ and $\operatorname{Prob}\left[a_{j, k}=1\right]=\lambda_{j}, j=1,2$. The joint pgf $A\left(z_{1}, z_{2}\right)$ of the number of arrivals of both classes is given by

$$
A\left(z_{1}, z_{2}\right)=1-\lambda_{1}-\lambda_{2}+q_{12}+\left(\lambda_{1}-q_{12}\right) z_{1}+\left(\lambda_{2}-q_{12}\right) z_{2}+q_{12} z_{1} z_{2}
$$

with $q_{12}$ a parameter. The correlationfactor $\rho_{a_{1} a_{2}}$ is given by

$$
\rho_{a_{1} a_{2}}=\frac{q_{12}-\lambda_{1} \lambda_{2}}{\sqrt{\lambda_{1} \lambda_{2}\left(1-\lambda_{1}\right)\left(1-\lambda_{2}\right)}}
$$

By varying $q_{12}$, the correlationfactor can be varied, while keeping the arrival rates of both classes constant ( $\lambda_{1}$ and $\lambda_{2}$ respectively). In Figure 13, we show the mean delay of class 2 packets versus the total load, for $\beta_{1}=\beta_{2}=0.5$ and $\alpha=0.25,0.5$ and 0.75 respectively. For the three cases, we vary the correlationfactor from its minimal value $\left(q_{12}=0\right)$ to zero $\left(q_{12}=\lambda_{1} \lambda_{2}\right)$ to its maximal value $\left(q_{12}=\max \left(\lambda_{1}, \lambda_{2}\right)\right)$. We see that the influence of the correlationfactor is limited for negative correlationfactors, while it is significant for positive correlationfactors, especially when the total load 
is high and the number of class 1 packets in the overall traffic mix is large. The reason for the higher mean class 2 delay for positive correlation between the number of arrivals of both classes is the increasing probability that in the arrival slot of a particular class 2 packet a class 1 packet arrives as well. The class 2 packet is served after this class 1 packet, thus increasing its mean delay.

[Figure 13 about here.]

\subsection{Comparing with continuous-time results}

In this subsection, we briefly compare our discrete-time results with continuous-time results. The slots in the discrete-time case are of length $T$. In the continuous-time case, we assume a Poisson arrival process for both classes, i.e., the number of arrivals of class $j(j=1,2)$ during a time-period $T$ is exponential and its pgf is given by $e^{\lambda_{j} T(z-1)}, j=1,2$ respectively. The same arrival process is assumed in the discretetime case, i.e., the joint generating function of the number of both classes during a slot is given by $e^{\left(\lambda_{1}+\lambda_{2}\right) T(z-1)}$. The service process for both classes (high and low priority) is the same. In the continuous-time case it is assumed to be exponentially distributed with departure rate $\mu$. In the discrete-time case, it is assumed to be geometrically distributed with parameters $\beta_{j}$ given by $1-\mu T$ (thus the service rate is also $\mu$ in the discrete-time queue). Summarizing, the arrival process is identical in both cases, while there are some differences in the service processes. Firstly, in the continuous case, more departures can occur in one slot, while this is not the case in the discrete-time system. Secondly, in the discrete-time queue, a packet can not begin service in its departure slot, while this is possible in the continuous-time system. So, we will expect that both systems will converge when $T$ goes to zero, while big differences are to be expected when $T$ is rather big.

To illustrate this, we show in Figure 14 the mean delay of both priority classes versus the slot length $\mathrm{T}$ in both continuous-time (results obtained from [9]) and discrete-time (results from this paper), when $\lambda_{1}=\lambda_{2}=0.08 / \mathrm{s}$ and $\mu=0.2 / \mathrm{s}$. For $T=0$, both the continuous-time as the discrete-time result is the same as expected. The continuous time results are independent of the slot length $T$, while the mean delay of both classes decreases with increasing $T$. This is mainly because of the decreasing variance of the service time with increasing $T$. Indeed, the variance of the (geometrical) service times is equal to $\frac{1-\mu T}{(\mu T)^{2}}$, and thus decreases with increasing $T$. When $T$ reaches $1 / \mu$, the variance reaches 0 , which means that the service times become deterministically equal to $T$. We see quite a big difference for the low priority 
delay for high $T$, as expected.

[Figure 14 about here.]

\section{Conclusion}

In this paper, we have analyzed a discrete-time GI-Geo-1 queue with a preemptive resume priority scheduling discipline and two priority classes. We have derived the joint generating function of the system contents of both classes and the generating functions of the packet delay of both classes. These pgf's are not explicitly found, but we have shown that the moments and the (approximate) tail probabilities of the system contents and packet delay can be found explicitly in terms of the system parameters. We have finally discussed the impact of priority scheduling on the performance characteristics by an extensive number of examples.

\section{Appendix 1 : Calculation of the Probability Mass Func- tion}

Given a generating function $X(z) \triangleq \sum_{n=0}^{\infty} x(n) z^{n}$, the question is how to find an explicit, practically usable expression for its corresponding pmf $x(n)$. From the definition of $X(z)$ it follows that $x(n)$ is the coefficient of $z^{n}$ in the expansion of $X(z)$ about $z=0$, or equivalently the coefficient of $z^{-1}$ in the expansion of $z^{-1-n} X(z)$ about $z=0 . x(n)$ is thus by definition the residue of the function $z^{-1-n} X(z)$ in the point $z=0$. Since the multiplicity of the pole $z=0$ of $z^{-1-n} X(z)$ depends linearly on $n$, calculating the residue in $z=0$ is nearly impossible for large $n$ (since evaluating the residue in an $k$-multiple pole requires $k$ derivations). Using the residue theorem of Cauchy however, it is proven that

$$
\begin{aligned}
x(n) & =\operatorname{Res}_{z=0}\left[X(z) z^{-1-n}\right] \\
& =\frac{1}{2 \pi i} \oint_{C_{1}} X(z) z^{-1-n} d z-\sum_{j=0}^{m} \operatorname{Res}_{z=z_{j}} X(z) z^{-1-n}
\end{aligned}
$$

with $i=\sqrt{-1}, C_{1}$ a contour with infinite radius and $z_{j}$ the poles of $X(z)$. The contour integral in the former expression is normally easy to calculate (in most cases the term equals zero). The sum of residues can be approximated by the residue in the dominant pole of $X(z)$. As a result, an easy, practically usable formula to calculate approximate tail probabilities is obtained. 


\section{Appendix 2 : Inversion of $(1-z)^{\alpha}$}

Theorem 1 Assume that, with the sole exception of the singularity $z=1$,

$$
F(z) \triangleq \sum_{n=1}^{\infty} f(n) z^{n}
$$

is analytic in the domain

$$
\Delta=\{z:|z| \leq 1+\eta,|\operatorname{Arg}(z-1)| \geq \theta\} \backslash\{1\},
$$

in which $\eta$ is a positive real number and $0<\theta<\pi / 2$. Assume further that as $z$ tends to 1 in $\Delta$,

$$
F(z)=K(1-z)^{\alpha},
$$

with $\alpha \notin \mathbb{N}$. Then, as $n \rightarrow \infty$,

$$
f(n)=\frac{K}{\Gamma(-\alpha)} n^{-\alpha-1}
$$




\section{References}

[1] H. Bruneel and B.G. Kim, Discrete-time models for communication systems including ATM, Kluwer Academic Publishers, Boston, 1993.

[2] H. Bruneel, B. Steyaert, E. Desmet and G.H. Petit, Analytic derivation of tail probabilities for queue lengths and waiting times in ATM multiserver queues, European Journal of Operational Research 76 (3) (1994) 563-572.

[3] I. Cidon and R. Guérin, On protective buffer policies, in: Proceedings of Infocom '93, San Francisco, 1993, pp. 1051-1058.

[4] M. Drmota, Systems of functional equations, Random Structures \& Algorithms 10 (1-2) (1997) 103-124.

[5] D. Fiems, B. Steyaert, H. Bruneel, Analysis of a discrete-time GI-G-1 queueing model subjected to bursty interruptions, Computers and Operations Research 30 (1) (2002) 139-153.

[6] P. Flajolet and A. Odlyzko, Singularity analysis of generating functions, SIAM Journal on discrete mathematics 3(2) (1990) 216-240.

[7] N.K. Jaiswal, Priority queues, Academic Press, New York, 1968.

[8] A. Khamisy and M. Sidi, Discrete-time priority queues with two-state Markov modulated arrivals, Stochastic Models 8 (2) (1992) 337-357.

[9] L. Kleinrock, Queueing systems volume II: Computer applications, John Wiley \& Sons, 1976.

[10] K. Laevens and H. Bruneel, Discrete-time multiserver queues with priorities, Performance Evaluation 33 (4) (1998) 249-275.

[11] K. Liu, D.W. Petr, V.S. Frost, H. Zhu, C. Braun and W.L. Edwards, Design and analysis of a bandwidth management framework for ATM-based broadband ISDN, IEEE Communications Magazine 35 (5) (1997) 138-145.

[12] F. Machihara, A bridge between preemptive and non-preemptive queueing models, Performance Evaluation 23 (1995) 93-106.

[13] R.G. Miller, Priority queues, Annals of Mathematical Statistics (1960) 86-103.

[14] S.P. Morgan, Queueing disciplines and passive congestion control in byte-stream networks, IEEE Transactions on Communications 39 (7) (1991) 1097-1106.

[15] D. Sandhu and M.J.M. Posner, A priority M/G/1 queue with application to voice/data communication, European Journal of Operational Research 40 (1989) 99-108. 
[16] L. Takacs, Priority queues, Operations Research 12 (1964) 63-74.

[17] H. Takagi, Queueing analysis A foundation of Performance Evaluation Volume 1: Vacation and priority systems, North-Holland, 1991.

[18] T. Takine and T. Hasegawa, The workload in the MAP/G/1 queue with statedependent services: its application to a queue with preemptive resume priority, Stochastic Models 10 (1) (1994) 183-204.

[19] T. Takine, B. Sengupta and T. Hasegawa, An analysis of a discrete-time queue for broadband ISDN with priorities among traffic classes, IEEE Transactions on Communications 42 (2-4) (1994) 1837-1845.

[20] J. Walraevens, B. Steyaert and H. Bruneel, Performance analysis of a singleserver ATM queue with a priority scheduling, Computers and Operations Research, to appear.

[21] J. Walraevens, B. Steyaert and H. Bruneel, Delay characteristics in discretetime GI-G-1 queues with non-preemptive priority queueing discipline, Performance Evaluation 50 (1) (2002) 53-75. 


\section{List of Figures}

1 Mean system contents versus the total load when the fraction of the class 1 load equals $0.25,0.5$ and $0.75 \ldots \ldots \ldots \ldots$

2 Variance of the system contents versus the total load when the fraction of the class 1 load equals $0.25,0.5$ and $0.75 \ldots \ldots \ldots 29$

3 Mean packet delay versus the total load when the fraction of class 1 load equals $0.25,0.5$ and $0.75 \ldots \ldots \ldots \ldots$

4 Variance of the packet delay versus the total load when the fraction of class 1 load equals $0.25,0.5$ and $0.75 \ldots \ldots \ldots$

5 Mean packet delay versus the mean service time of class 2 packets when the fraction of class 1 load equals $0.25,0.5$ and $0.75 \ldots \ldots$

6 Mean packet delay versus the mean service time of class 1 packets when the fraction of class 1 load equals $0.25,0.5$ and $0.75 \ldots 33$

$7 \quad$ Mean packet delay of both classes versus the number of inlets . . . . 34

8 Regions for tail behavior as a function of the load of both classes . . 35

9 Tail behavior of the high and low priority cell delay for some combinations of class 1 and class 2 arrival rates . . . . . . . . . 36

10 Maximum load versus the fraction of class 1 load for several values of

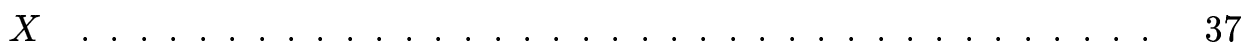

11 Maximum load versus the fraction of class 1 load for several values of

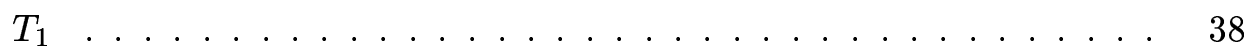

12 Maximum load versus the fraction of class 1 load for several values of

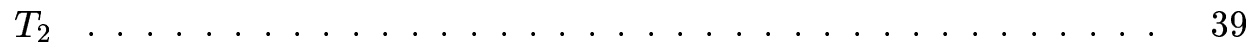

13 Mean packet delay of class 2 versus the total load when the fraction of class 1 arrivals equals $0.25,0.5$ and 0.75 and with different $\rho_{a_{1} a_{2}}$.

14 Comparison of the mean packet delay of both classes versus the slow length in continuous-time and discrete-time ......... 


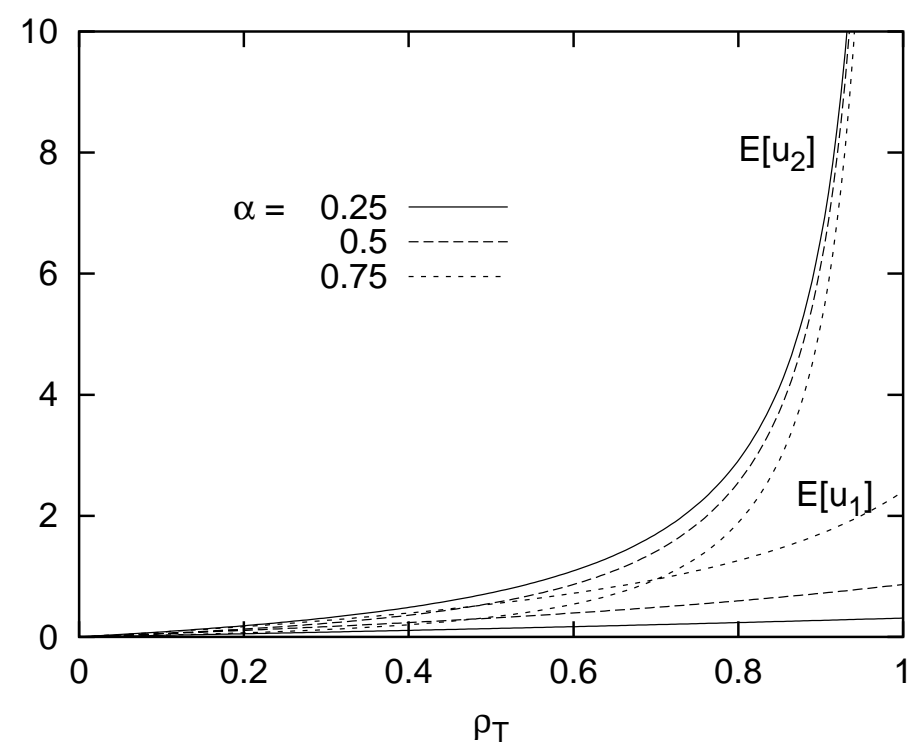

Figure 1: Mean system contents versus the total load when the fraction of the class 1 load equals $0.25,0.5$ and 0.75 


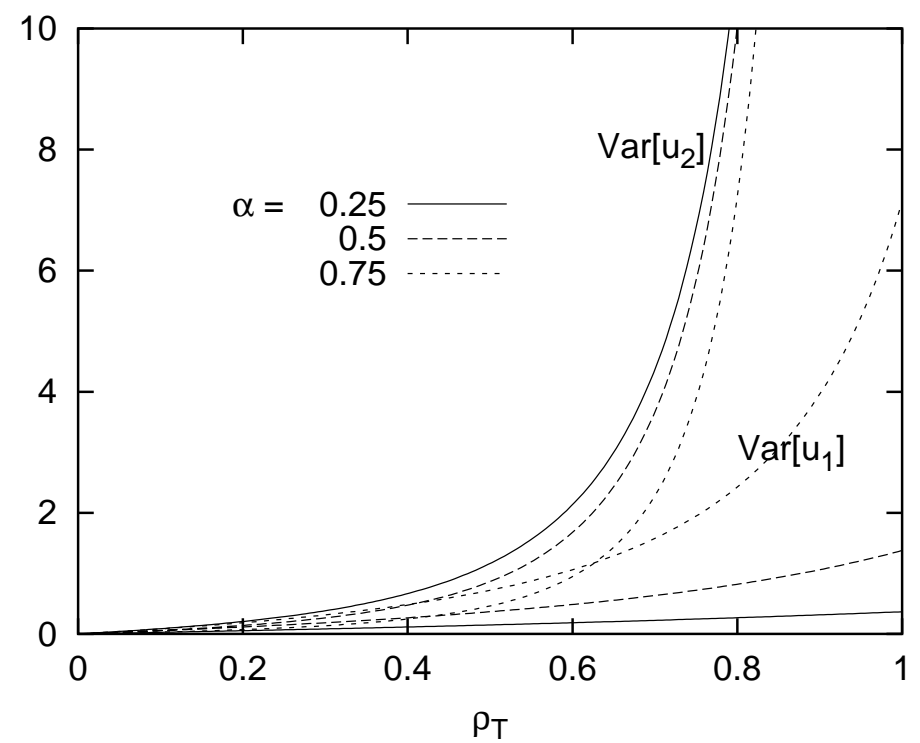

Figure 2: Variance of the system contents versus the total load when the fraction of the class 1 load equals $0.25,0.5$ and 0.75 


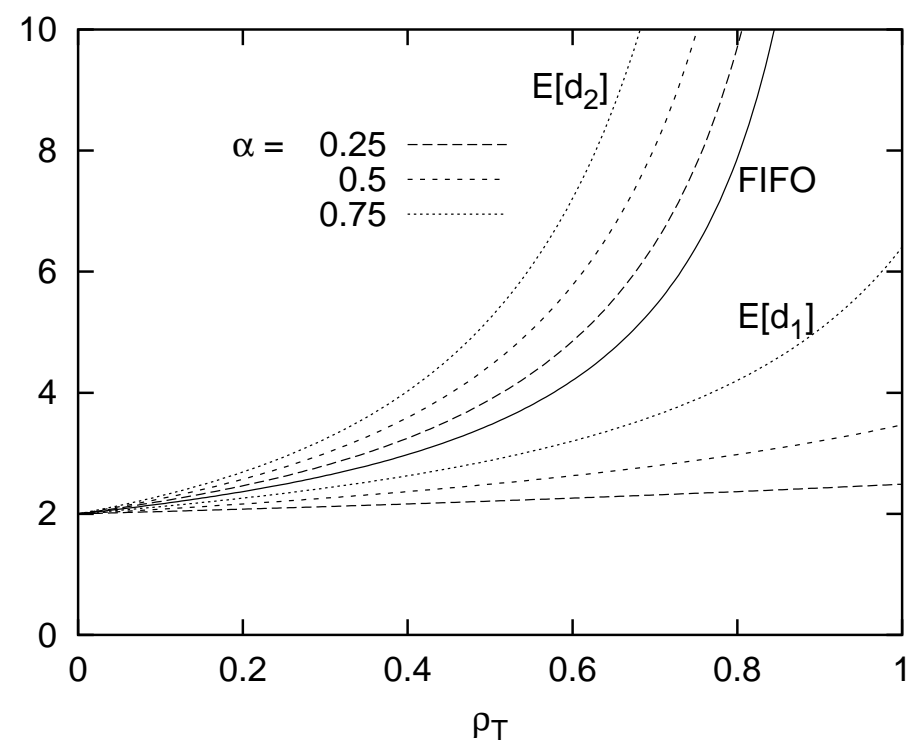

Figure 3: Mean packet delay versus the total load when the fraction of class 1 load equals $0.25,0.5$ and 0.75 


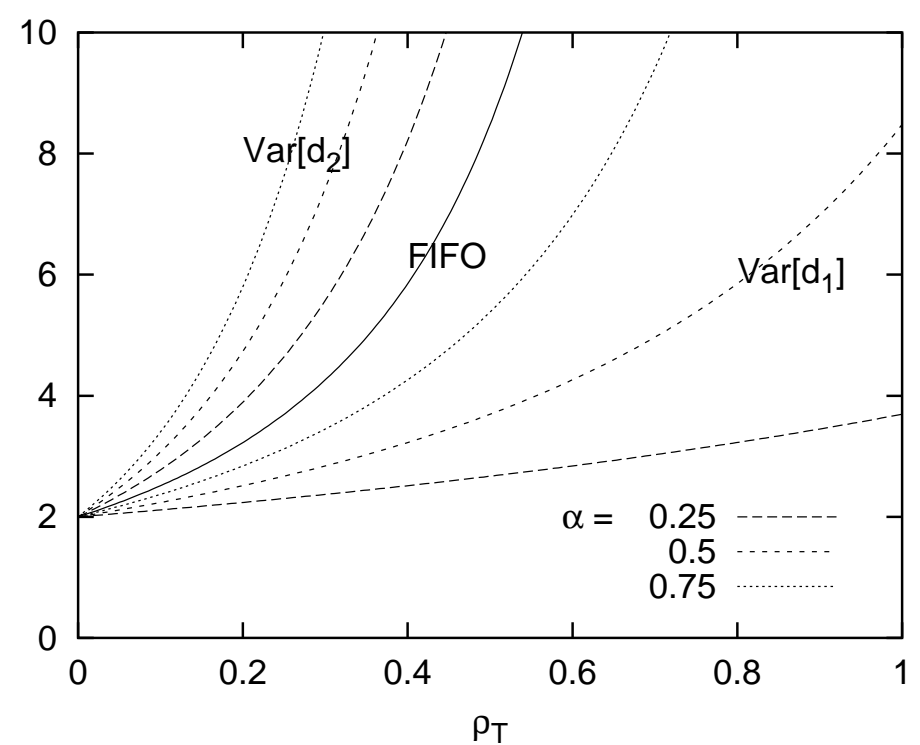

Figure 4: Variance of the packet delay versus the total load when the fraction of class 1 load equals $0.25,0.5$ and 0.75 


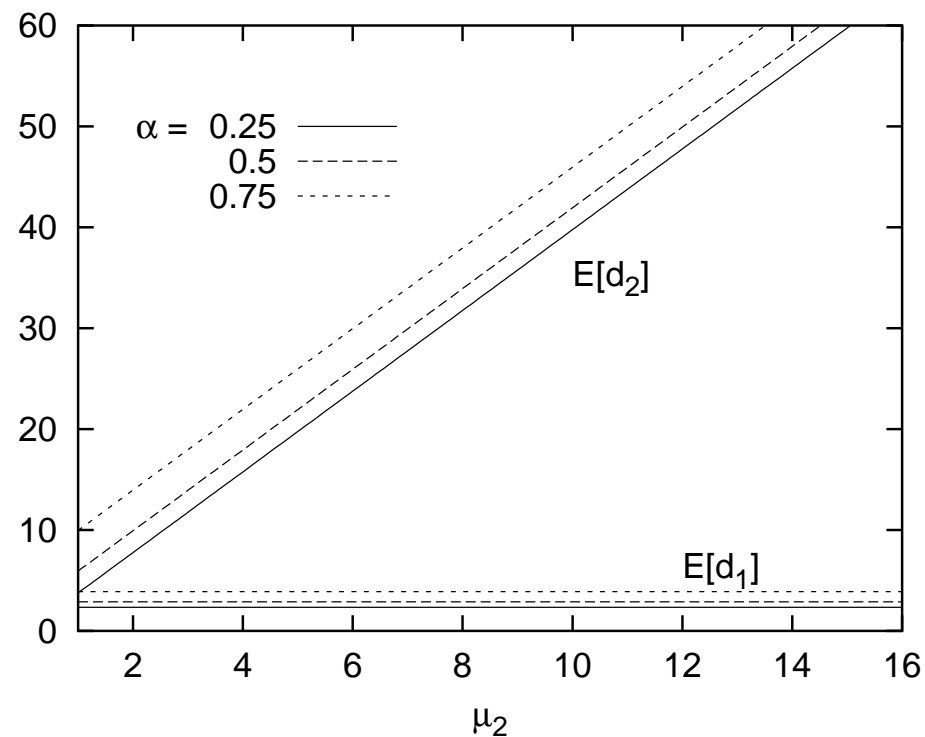

Figure 5: Mean packet delay versus the mean service time of class 2 packets when the fraction of class 1 load equals $0.25,0.5$ and 0.75 


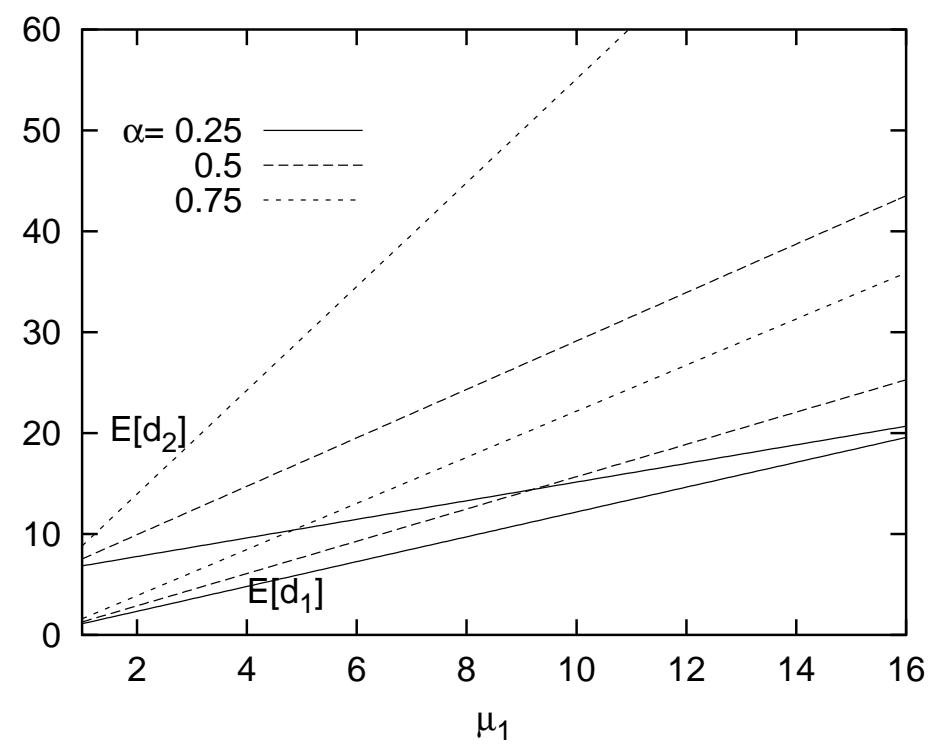

Figure 6: Mean packet delay versus the mean service time of class 1 packets when the fraction of class 1 load equals $0.25,0.5$ and 0.75 


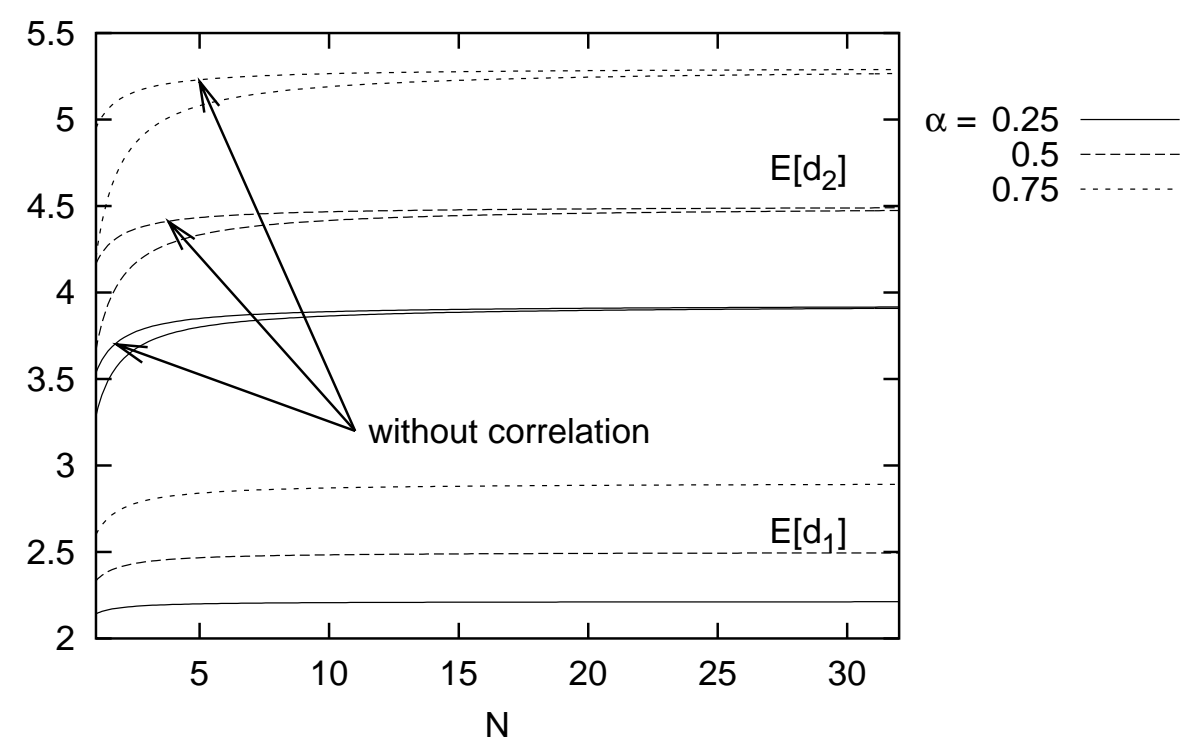

Figure 7: Mean packet delay of both classes versus the number of inlets 


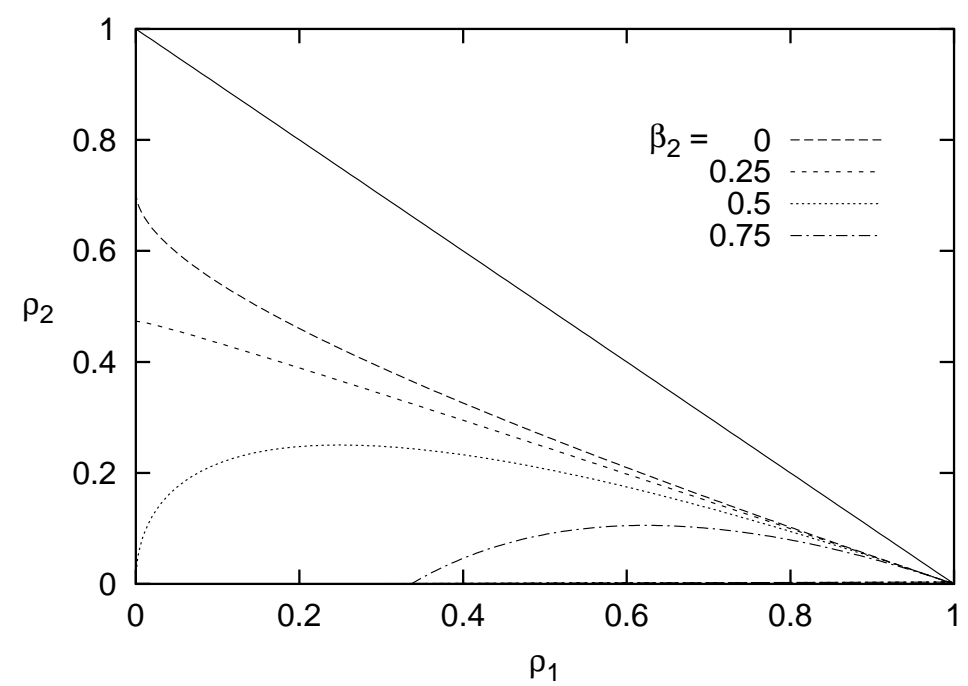

Figure 8: Regions for tail behavior as a function of the load of both classes 


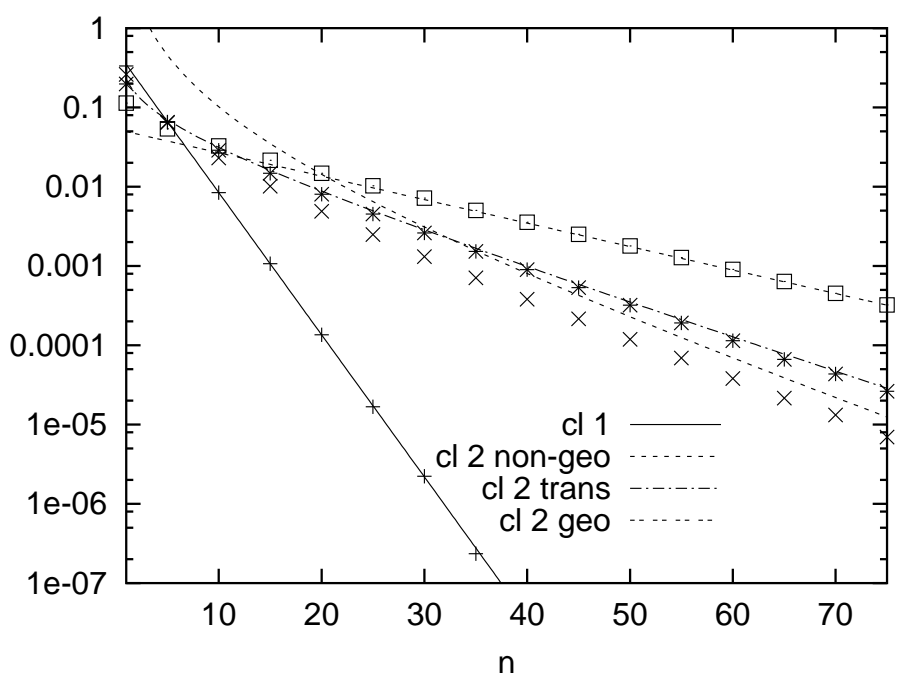

Figure 9: Tail behavior of the high and low priority cell delay for some combinations of class 1 and class 2 arrival rates 


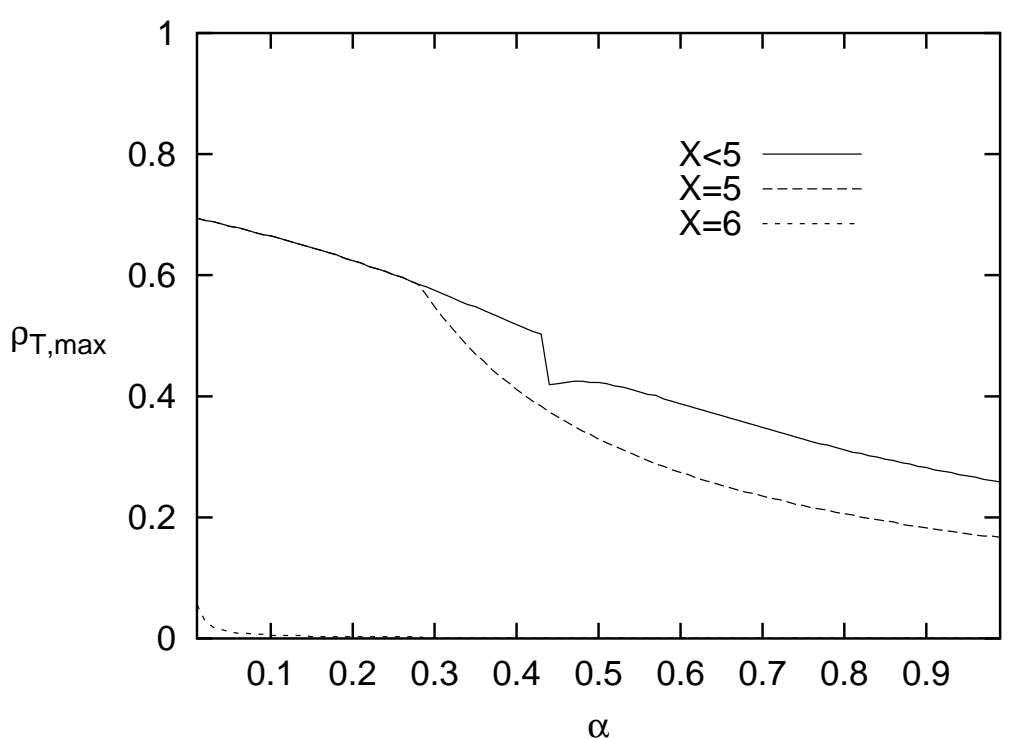

Figure 10: Maximum load versus the fraction of class 1 load for several values of $X$ 


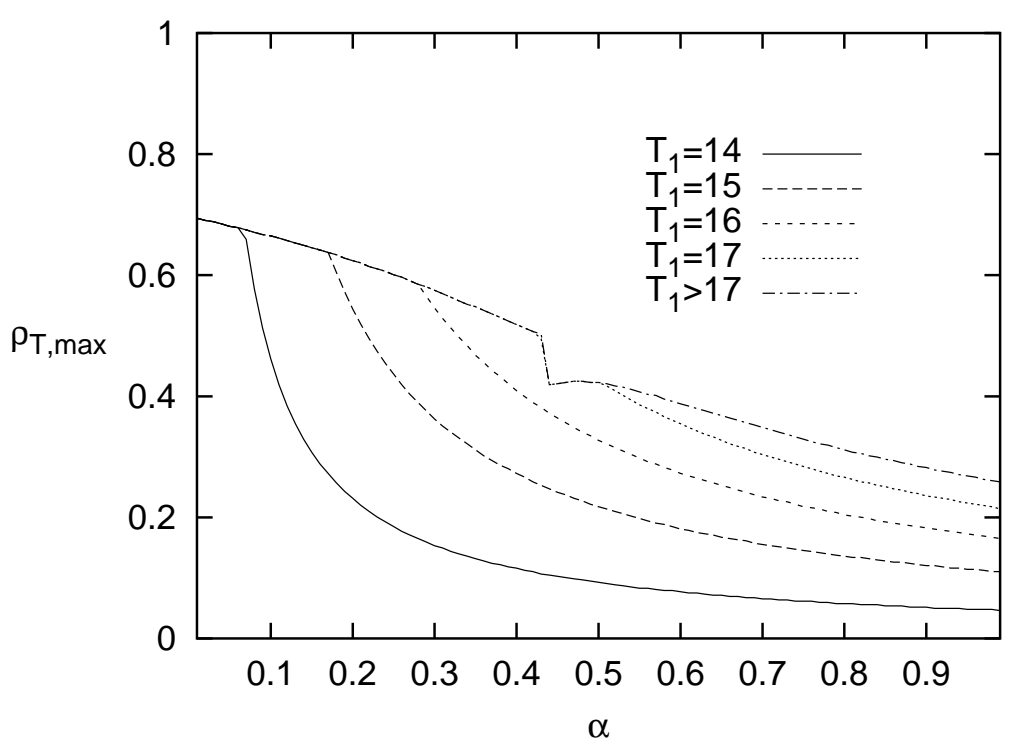

Figure 11: Maximum load versus the fraction of class 1 load for several values of $T_{1}$ 


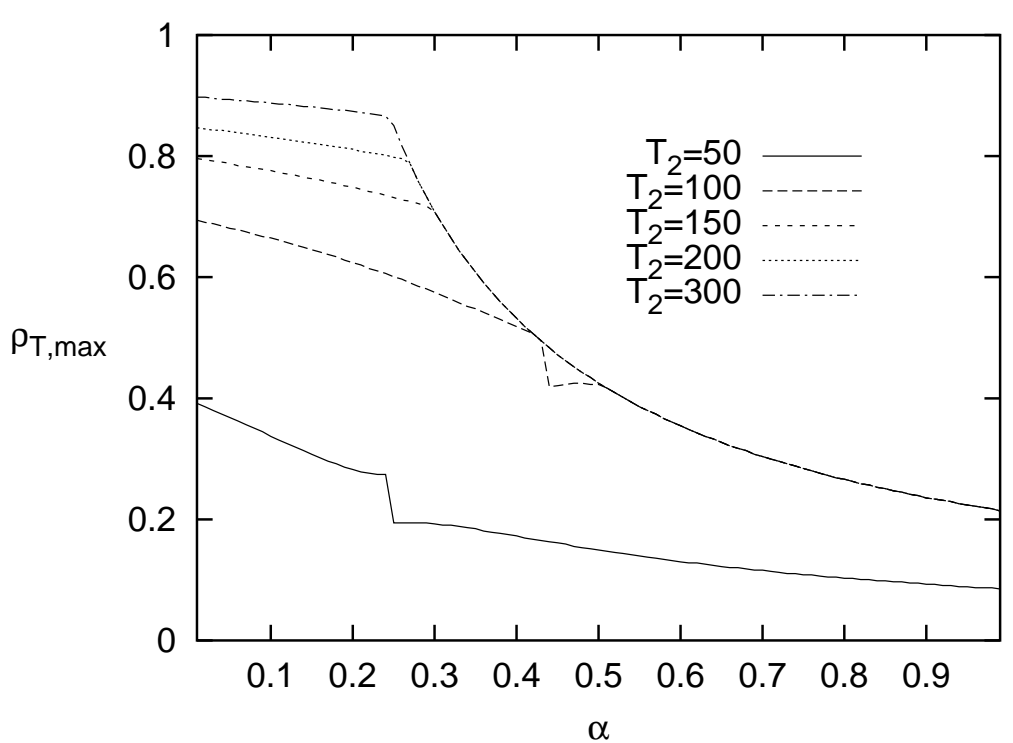

Figure 12: Maximum load versus the fraction of class 1 load for several values of $T_{2}$ 


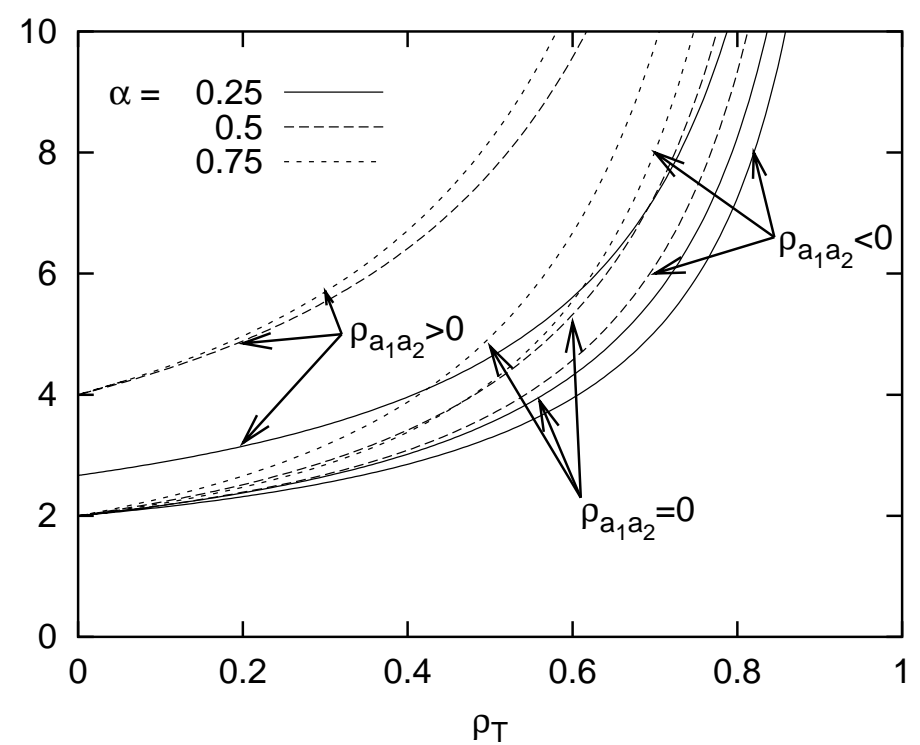

Figure 13: Mean packet delay of class 2 versus the total load when the fraction of class 1 arrivals equals $0.25,0.5$ and 0.75 and with different $\rho_{a_{1} a_{2}}$ 


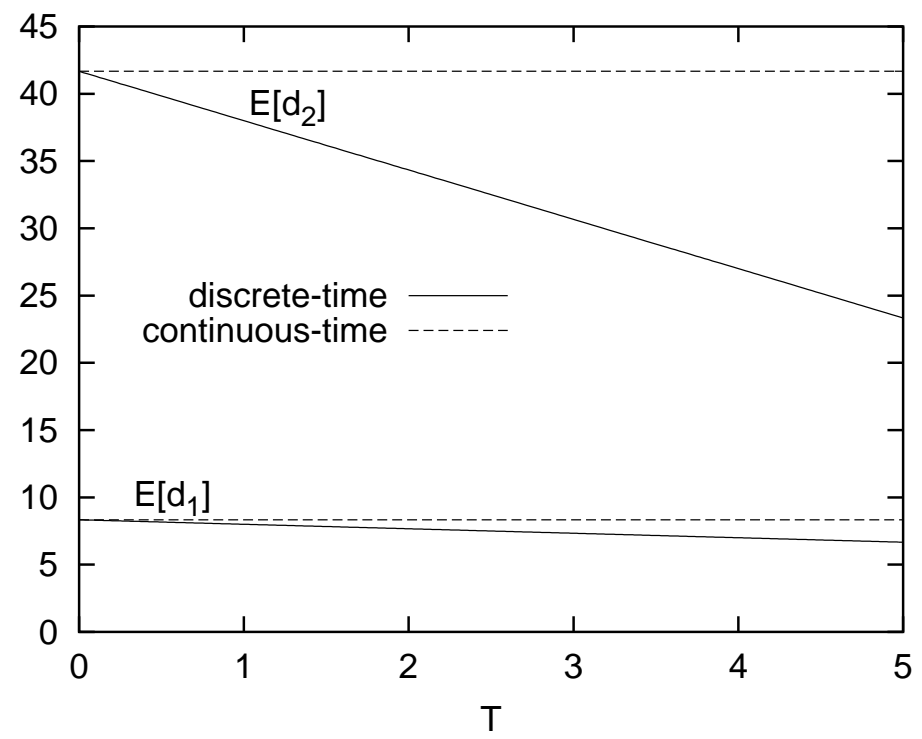

Figure 14: Comparison of the mean packet delay of both classes versus the slow length in continuous-time and discrete-time 